\title{
LOCAL DECAY OF SOLUTIONS OF CONSERVATIVE FIRST ORDER HYPERBOLIC SYSTEMS IN ODD DIMENSIONAL SPACE(1)
}

\author{
BY
}

\author{
JAMES V. RALSTON
}

\begin{abstract}
This paper deals with symmetric hyperbolic systems, $\partial u / \partial t=L u$, where $L$ is equal to the homogeneous, constant coefficient operator $L_{0}$ for $|x|>R$. Under the hypothesis that $L$ has simple null bicharacteristics and these propagate to infinity, local decay of solutions and completeness of the wave operators relating solutions of $\partial u / \partial t$ $=L u$ and solutions of $\partial u / \partial t=L_{0} u$ are established. Results of this type for elliptic $L$ are due to Lax and Phillips. The proof here is based, in part, on a new estimate of the regularity of the $L^{2}$-solutions of the equation $L u+(i \lambda+\varepsilon) u=g$ for smooth $g$ with support in $|x| \leq R$.
\end{abstract}

Let $L$ be an operator of the form

$$
L=\sum_{j=1}^{n} A_{j}(x) \frac{\partial}{\partial x_{j}}+B(x) .
$$

The coefficients $A_{j}(x)$ are $k \times k$ hermitian matrices. They are smooth functions of $x$ on $\mathbf{R}^{n}$ and take constant values $A_{j}^{0}$ for $|x|>R$. The matrix $B(x)$ is also a smooth function of $x$ and it vanishes for $|x|>R$. $B(x)$ is chosen so that $L$ is formally skew-hermitian. Under these hypotheses the graph closure of $L$ defined on $C_{0}^{\infty}\left(\mathbf{R}^{n}\right)$ is a skew-hermitian operator on $L^{2}\left(\mathbf{R}^{n}\right) . C_{0}^{\infty}\left(\mathbf{R}^{n}\right)$ and $L^{2}\left(\mathbf{R}^{n}\right)$ denote the spaces of $\mathbf{C}^{k}$-valued smooth functions of compact support and $\mathbf{C}^{k}$-valued square-integrable functions respectively. The operator

$$
L_{0}=\sum_{j=1}^{n} A_{j}^{0} \frac{\partial}{\partial x_{j}}
$$

is also skew-hermitian on $L^{2}\left(\mathbf{R}^{n}\right)$. Thus $L$ and $L_{0}$ generate unitary groups $U(t)$ and $U_{0}(t)$ on $L^{2}\left(\mathbf{R}^{n}\right)$, and the solutions to the initial value problems $u(0)=f_{1}$ $\in C_{0}^{\infty}\left(\mathbf{R}^{n}\right), \partial u / \partial t=L u$, and $u(0)=f_{2} \in C_{0}^{\infty}\left(\mathbf{R}^{n}\right), \partial u / \partial t=L_{0} u$, are given by $U(t) f_{1}$ and $U_{0}(t) f_{2}$.

In this paper we are concerned with the local decay of solutions to the hyperbolic system of equations $\partial u / \partial t=L u$. Defining

Received by the editors December 20, 1972 and, in revised form, June 8, 1973.

AMS (MOS) subject classifications (1970). Primary 35L45; Secondary 35P25, 35P10.

(1) Research work in the preparation of this paper was supported by the Office of Naval Research under Contract N000-14-69-A-0200-4022, Contract Authority Identification No. 044-144. Reproduction in whole or in part is permitted for any purpose of the United States Government. 


$$
\|f\|^{r}=\left(\int_{|x|<r}|f|^{2} d x\right)^{1 / 2}
$$

for $f \in L^{2}\left(\mathbf{R}^{n}\right)$, we say a solution with initial data $f$ decays locally if $\lim _{t \rightarrow \pm \infty}\|U(t) f\|^{r}=0$ for all $r>0$. From the standpoint of scattering, the desirable result is that local decay holds for all initial data orthogonal to $H_{0}$, the subspace of $L^{2}\left(\mathbf{R}^{n}\right)$ spanned by the eigenvectors of $L$.

From the theory of hyperbolic equations one might suspect that local decay would depend on the behavior of the bicharacteristic curves of $\partial / \partial t-L$, or, equivalently, the bicharacteristic curves of $L$. However, Lax and Phillips [6] have shown if $L$ is elliptic one always has local decay on $L^{2}\left(\mathbf{R}^{n}\right) \ominus H_{0}$. The results we present here indicate it is the behavior of the null bicharacteristics of $L$ (these are absent when $L$ is elliptic) which influences the local decay of solutions. Our result is the following

Theorem I. Let $A(x, \xi)=\sum_{j=1}^{n} A_{j}(x) \xi_{j},(x, \xi) \in \mathbf{R}^{n} \times \mathbf{R}^{n}$.

Assume the following:

(i) The null space of $A(x, \xi)$ is at most one dimensional for $\xi \neq 0$. This implies there is a smooth function $\tau(x, \xi)$, defined on a conic neighborhood of $S=\{(x, \xi) \mid$ $\operatorname{det} A(x, \xi)=0,|\xi|=1\}$, and vanishing on $S$, such that $\operatorname{det}(A(x, \xi)-\tau(x, \xi) I)$ $=0$.

(ii) Given $\left(x_{0}, \xi_{0}\right) \in S \cap\{(x, \xi)|| x \mid \leq R\}$ and $r>0$ there is a $T$ such that the bicharacteristic curve $(x(t), \xi(t)), \dot{x}(t)=-(\partial / \partial \xi) \tau(x, \xi), \dot{\xi}(t)=(\partial / \partial x) \tau(x, \xi), x(0)$ $=x_{0}, \xi(0)=\xi_{0}$ satisfies $|x(t)|>r$ for $|t|>T$.

(iii) $n$ is odd.

Under these assumptions:

(a) The point spectrum of $L$ has no accumulation points and with the possible exception of zero has finite multiplicity.

(b) Given $f \in L^{2}\left(\mathbf{R}^{n}\right)$ there exist $g_{ \pm} \in L^{2}\left(\mathbf{R}^{n}\right) \ominus H_{0}$ such that

$$
\lim _{t \rightarrow \pm \infty}\left\|U(t) g_{ \pm}-U_{0}(t) f\right\|=0
$$

and given $g \in L^{2}\left(\mathbf{R}^{n}\right) \ominus H_{0}$ there exist $f_{ \pm} \in L^{2}\left(\mathbf{R}^{n}\right)$ such that

$$
\lim _{t \rightarrow \pm \infty}\left\|U(t) g-U_{0}(t) f_{ \pm}\right\|=0 .
$$

(c) For all $f \in L^{2}\left(\mathbf{R}^{n}\right) \ominus H_{0}$ and $r>0$,

$$
\lim _{t \rightarrow \pm \infty}\|U(t) f\|^{r}=0
$$

One might hope to replace hypothesis (ii) by the assumption that the group velocity $|\partial \tau / \partial \xi|$ does not vanish on $S$, but Example 4.1 shows this assumption does not imply conclusions (b) or (c) (a slight modification of this example would show it does not imply (a) either). From the standpoint of applications some 
weakening of (i) is desirable.( $\left.{ }^{2}\right)$ For instance, the linearized equations of magnetohydrodynamics fail to satisfy (i). Hypothesis (iii) arises because of our use of the Radon transform in $\$ 2$ and in the proof of Corollary 1.3.( $\left.{ }^{3}\right)$ In view of recent results of Lax and Phillips [8] it is possible that (iii) is unnecessary. Conclusion (a) is probably much too weak: all known examples (cf. [11]) have no point spectrum except for $\lambda=0$ and this is an eigenvalue of finite multiplicity. For $L$ elliptic with the unique continuation property this is the general situation (cf. [7, Theorem 3.1]). Under hypotheses (i)-(ii) it is easy to show local decay holds for the group $U_{0}(t)$ on $L^{2}\left(\mathbf{R}^{n}\right)$. Hence (c) is an immediate consequence of the second part of (b). Conclusion (b) gives the existence and unitarity of the scattering operator relating $U(t)$ and $U_{0}(t)$.

Our proof of (b) is modeled on the proof used by Phillips and Sarason [9] to show the existence of the scattering operator in a boundary value problem for an elliptic $L$ with nonelliptic boundary conditions. We construct generalized (not square-integrable) eigenfunctions of $L$ satisfying a type of radiation condition and use these to give an explicit diagonalization of the restriction of $L$ to subspaces $\mathrm{H}^{+}$and $\mathrm{H}^{-}$. The generalized eigenfunctions are constructed by the limiting absorption method. This method was developed rigorously by Eidus [1] and has been widely used in scattering theory to treat elliptic problems and problems of constant deficit (cf. [2] and [10]). To use limiting absorption here we require an estimate which will take the place of the coercive estimates from elliptic theory. This estimate, Theorem 1.1, is proved in $\$ 1$ and makes essential use of recent work of Hörmander [5]. In $\$ 2$ we construct the generalized eigenfunctions and the diagonalizations of $L$ on $H_{+}$and $H_{-}$. We also prove a crucial preliminary decay result, Theorem 2.2 . In $\$ 3$ we complete the program of [9] by showing $H_{+}+H_{-}$is dense in $L^{2}\left(\mathbf{R}^{n}\right) \ominus H_{0}$. In doing this we make use of an operator-valued function $Q(t)$ which approximately commutes with $\partial / \partial t-L$. The construction of this operator was suggested by the construction in $\$ 2.4$ of [3].

In the final section, $\S 4$, we use $Q(t)$ again to show that the behavior of the nonnull bicharacteristics of $L$ does influence the uniformity of the rate of local decay. We show that if $L$ has a simple bicharacteristic which remains in $|x|<R$ as $t \rightarrow \infty$, then for all $t>0$ and $\varepsilon>0$ one can find initial data $f$ supported in $|x|<R$ such that $\|U(t) f\|^{R} \geq(1-\varepsilon)\|f\|$. We believe this result could be deduced directly from [4], but the proof given here is short and self-contained.

I am grateful to Thomas Beale, Andrew Majda, John Palmer, and Ralph Phillips for suggesting many improvements in a preliminary version of this work.

(2) Added in proof. This extension will require methods different from those used here. One can construct a $2 \times 2$ operator $L$ in two variables with diagonal top order symbol such that the bicharacteristics of $L$ propagate to infinity and the conclusion of Theorem 1.1 does not hold.

$\left({ }^{3}\right)$ Added in proof. Patrick Murphy has pointed out that Theorem 3.3 of [7] can be extended to the case of even $n$, simply by considering an operator in $n$ variables as an operator in $n+1$ variables with a vanishing coefficient. Hence, Corollary 1.3 (which is conclusion (a)) holds for even $n$. 
1. The coercive estimate. We begin by constructing functions to be used later and fixing notation. Let $S=\left\{(x, \xi) \in \mathbf{R}^{n} \times \mathbf{R}^{n}|\operatorname{det} A(x, \xi)=0, \quad| \xi \mid=1\right\}$, and let $N(x, \xi)$ be the null space of $A(x, \xi)$. By hypothesis $N(x, \xi)$ is one dimensional for $(x, \xi) \in S$. Hence, since $S_{R}=S \cap\{(x, \xi)|| x \mid \leq R\}$ is compact and $A(x, \xi)$ is independent of $x$ for $|x| \geq R$, there is a $\delta>0$ such that $A(x, \xi)-z I$ is invertible on $N(x, \xi)^{\perp}$ for $|z| \leq \delta,(x, \xi) \in S$. By continuity $R(x, \xi, z)=(A(x, \xi)-z I)^{-1}$ exists for $|z|=\delta$ for $(x, \xi)$ in an open neighborhood $U$ of $S$. We may assume $U \cap\left\{(x, \xi) \mid x=x_{0}\right\}$ is independent of $x_{0}$ for $\left|x_{0}\right| \geq R$ and each connected component of $U$ intersects $S$. We define a smooth projectionvalued function on $U$ by

$$
P(x, \xi)=\frac{-1}{2 \pi i} \oint_{|z|=\delta} R(x, \xi, z) d z .
$$

For $(x, \xi) \in U, A(x, \xi) P(x, \xi)=\tau(x, \xi) P(x, \xi)$, where $\tau(x, \xi)$ is the eigenvalue of $A(x, \xi)$ given by

$$
\tau(x, \xi)=\frac{-1}{2 \pi i} \oint_{|z|=\delta} z \operatorname{det} R \frac{\partial}{\partial z}\left(\operatorname{det} R^{-1}\right) d z .
$$

For $(x, \xi) \in S, P(x, \xi)$ is the orthogonal projection on $N(x, \xi)$ and $\tau(x, \xi)=0$. $P(x, \xi)$ and $\tau(x, \xi)$ are extended to a conic neighborhood $V \supset U$ via $P(x, \xi)$ $=P(x, \xi /|\xi|)$ and $\tau(x, \xi)=|\xi| \tau(x, \xi /|\xi|)$.

The chief objective of this section is to prove the following estimate in Sobolev norms ( $\|f\|_{0}^{r}$ is just the localized $L^{2}$-norm, $\|f\|^{r}$, introduced earlier):

Theorem 1.1. Let $L u+(i \lambda \pm \varepsilon) u=g, g \in C_{0}^{\infty}(|x|<R), u \in L^{2}\left(\mathbf{R}^{n}\right), 0<\varepsilon$ $\leq 1,|\lambda| \leq K$. Then there is an $R^{\prime}$ such that given $s$ there is a cutoff $\alpha \in C_{0}^{\infty}$, $\alpha(x)=1,|x| \leq R$, for which

$$
\|\alpha u\|_{s} \leq C_{s}\left(\|g\|_{s}+\|u\|_{0}^{R^{\prime}}\right) .
$$

$R^{\prime}$ and $C_{s}$ is independent of $\lambda, \varepsilon$, and $u$.

The main ingredient in the proof of Theorem 1.1 is the following estimate due to Hörmander:

Let $p(x, \xi)$ be a real symbol of order one such that $d p \neq 0$ on $p^{-1}(0)$, and let $\gamma(t)$ be a bicharacteristic strip for $p$ contained in $p^{-1}(0)$, such that the projection of $\gamma(t)$ onto $\{(x, \xi)|| \xi \mid=1\}$ is injective. Then, given $t_{1}, t_{2}$ and conic neighborhoods $N_{1}$ and $N_{2}$ of $\gamma\left(t_{1}\right)$ and $\left\{\gamma(t) \mid t_{1} \leq t \leq t_{2}\right\}$ respectively, there are symbols of order zero, $\rho, \rho_{1}$ and $\rho_{2}$, such that $\rho_{i}$ is supported in $N_{i}, i=1,2, \rho=1$, on a conic neighborhood of $\left\{\gamma(t) \mid t_{1} \leq t \leq t_{2}\right\}$ and

$$
\|\rho(x, D) u\|_{s} \leq C_{s, k}\left(\left\|\rho_{1}(x, D) u\right\|_{s}+\left\|\rho_{2}(x, D) p(x, D) u\right\|_{s}+\left\|\rho_{2}(x, D) u\right\|_{-k}\right)
$$

for any distribution $u$. 
This is Proposition 3.5.1 of [5] restricted to the case of real symbols and restated in quantitative rather than qualitative form (in [5] the conclusion is that $\rho u$ is in $H^{s}$ if $\rho_{1} u$ and $\rho_{2} p u$ are in $H^{s}$, but the proof of this yields the estimate given here). This is an analogue for operators with simple null bicharacteristics of the basic regularity estimates of elliptic theory.

Given a distribution solution $u$ to $L u+(i \lambda+\varepsilon) u=g$ we will prove Theorem 1.1 by using (1.1) together with elliptic estimates. However, this requires us to bound the regularity of $u$ (as measured by $\left\|\rho_{1}(x, D) u\right\|_{s}$ ) by a constant multiple of $\|g\|_{s}+\|u\|_{0}^{R^{\prime}}$ at some point $\gamma\left(t_{1}\right)$ on each null bicharacteristic emanating from $S_{R}$. By hypothesis (ii), given $\left(x_{0}, \xi_{0}\right) \in S_{R}$, there is a $T$ such that the bicharacteristic $(x(t), \xi(t))$ with

$$
d x / d t=-(\partial \tau / \partial \xi)(x, \xi), \quad d \xi / d t=(\partial \tau / \partial x)(x, \xi),
$$

$x(0)=x_{0}, \xi(0)=\xi_{0}$, satisfies $|x(t)|>R$ for $|t|>T$. Thus we may always choose $\gamma\left(t_{1}\right)=\left(x\left(t_{1}\right), \xi\left(t_{1}\right)\right)$ with $\left|x\left(t_{1}\right)\right|>R$. Even so, the desired bound does not hold for arbitrary solutions of $L u+(i \lambda+\varepsilon) u=g$. A suitable bound with constants independent of $\varepsilon$ does hold for the unique solution in $L^{2}\left(\mathbf{R}^{n}\right)$, as is shown by the following lemma.

Lemma 1.2. Let $(x(t), \xi(t))$ be the bicharacteristic described above. Then there is $a T_{0}>T$ and there are pseudo-differential operators $\varphi_{ \pm}(x, D)$ of order zero with symbols $\varphi_{ \pm}(x, \xi)=P(x, \xi)$ on conic neighborhoods $N_{ \pm}$of $\left(x\left( \pm T_{0}\right), \xi\left( \pm T_{0}\right)\right)$ for which the following holds: If $u$ is the square-integrable solution to $L u+(\lambda \mp i \varepsilon) u$ $=g, g \in C_{0}^{\infty}(|x|<R)$, then

$$
\left\|\varphi_{ \pm}(x, D) u\right\|_{s} \leq C_{s}\left(\|g\|_{0}+\|u\|_{0}^{R}\right) .
$$

Here $C_{s}$ is independent of $\varepsilon$ and $\lambda$ for $0<\varepsilon \leq 1$ and $|\lambda|<K$.

Proof. We assume $L u+(i \lambda+\varepsilon) u=g$. The proof for $L u+(i \lambda-\varepsilon) u=g$ is identical.

Set $(\hat{x}, \hat{\xi})=(x(-T), \xi(-T))$ and set $P(\xi)=P(x, \xi), \tau(\xi)=\tau(x, \xi)$ for $|x|$ $>R$. By hypothesis (ii), $\partial \tau / \partial \xi(\hat{\xi}) \neq 0$. We rotate coordinates in $\xi$-space by a matrix $0, \xi^{\prime}=0 \xi$ so that $\xi^{\prime}=(1,0, \ldots, 0)$ is normal to the surface $\tau(\xi)=0$ at $\xi=\hat{\xi}$ and $\partial \tau \circ 0^{*} / \partial \xi_{1}^{\prime}(0 \hat{\xi})>0$. Next we make a second change of coordinates $\eta=\Lambda\left(\xi^{\prime}\right)=\left(\tau\left(0^{*} \xi^{\prime}\right), \xi_{2}^{\prime}, \ldots, \xi_{n}^{\prime}\right)$. The jacobian of $\Lambda\left(\xi^{\prime}\right), \Lambda^{\prime}\left(\xi^{\prime}\right)$, is homogeneous degree zero and hence $\operatorname{det} \Lambda^{\prime}\left(\xi^{\prime}\right) \neq 0$ on a conic neighborhood of $0 \hat{\xi}=\hat{\xi}^{\prime}$. Suppose there is no conic neighborhood of $\hat{\xi}^{\prime}$ on which $\Lambda\left(\xi^{\prime}\right)$ is injective. Then there are pairs $\rho_{m}^{1}, \rho_{m}^{2}$ such that $\rho_{m}^{i} /\left|\rho_{m}^{i}\right| \rightarrow \hat{\xi}^{\prime} /\left|\hat{\xi}^{\prime}\right|$ as $m \rightarrow \infty, i=1,2$, and $\Lambda\left(\rho_{m}^{1}\right)=\Lambda\left(\rho_{m}^{2}\right)$. Since $\hat{\xi}^{\prime} \cdot \partial \tau \circ 0^{*} / \partial \xi^{\prime}\left(\hat{\xi}^{\prime}\right)=0, \hat{\xi}^{\prime}=\left(0, \hat{\xi}_{2}^{\prime}, \ldots, \hat{\xi}_{n}^{\prime}\right) \neq 0$. Hence $\lim _{m \rightarrow \infty}\left|\rho_{m}^{1}\right| /\left|\rho_{m}^{2}\right|=1$, and now $\Lambda\left(\left|\hat{\xi}^{\prime}\right| \rho_{m}^{1} /\left|\rho_{m}^{1}\right|\right)=\Lambda\left(\left|\hat{\xi}^{\prime}\right| \rho_{m}^{2} /\left|\rho_{m}^{1}\right|\right)$ leads to a contradiction to the injectivity of $\Lambda\left(\xi^{\prime}\right)$ on an ordinary neighborhood of $\hat{\xi}^{\prime}$. Thus, defining $I(\xi)=\Lambda(0 \xi), I(\xi)$ is a diffeomorphism of a conic neighborhood $C$ of $\hat{\xi}$ onto a conic neighborhood $\tilde{C}$ of $I(\hat{\xi})$. Let $\varphi(\xi)$ be a symbol of order zero such 
that $\varphi(\xi)=1$ on a conic neighborhood of $\hat{\xi}$ and support $\varphi$ is contained in $C$. Define $q(\xi)=\varphi(\xi) P(\xi)$, and let $Q(D)$ be the pseudo-differential operator with symbol $q(\xi)$.

By hypothesis $u \in L^{2}\left(\mathbf{R}^{n}\right)$, and in the sense of distributions

$$
L_{0} u+(i \lambda+\varepsilon) u=g+\left(L_{0}-L\right) u=h .
$$

Note support $h \subset|x| \leq R$. Taking fourier transforms and multiplying by $q(\xi)$,

$$
i \tau(\xi) q(\xi) \hat{u}(\xi)+(i \lambda+\varepsilon) q(\xi) \hat{u}(\xi)=q(\xi) \hat{h}(\xi) .
$$

Hence

$$
Q(D) u=\mathscr{F}^{-1} \frac{q(\xi) \hat{h}(\xi)}{i(\tau(\xi)+\lambda)+\varepsilon}
$$

Let $\hat{h}_{N}(\xi)=\psi(|\xi| / N) \hat{h}(\xi)$ and $\hat{u}_{N}=\psi(|\xi| / N) \hat{u}(\xi)$ where $\psi \in C_{0}^{\infty}(\mathbf{R})$ and $\psi(s)$ $=1$ for $|s|<1$. Then

$$
\begin{aligned}
\left(Q(D) u_{N}\right)(x) & =(2 \pi)^{-n / 2} \int e^{i x \cdot \xi} \frac{q(\xi) \hat{h}_{N}(\xi)}{i(\tau(\xi)+\lambda)+\varepsilon} d \xi \\
& =(2 \pi)^{-n / 2} \int \exp \left(i x \cdot I^{-1}(\eta)\right) \frac{\hat{H}_{N}(\eta)}{i\left(\eta_{1}+\lambda\right)+\varepsilon} d \eta
\end{aligned}
$$

where $\hat{H}_{N}(\eta)=q\left(I^{-1}(\eta)\right) \hat{h}_{N}\left(I^{-1}(\eta)\right)\left(\operatorname{det}\left(I^{-1}\right)^{\prime}(\eta)\right)$. Thus

$$
Q(D) u_{N}(x)=(2 \pi)^{-n} \int \exp \left(i x \cdot I^{-1}(\eta)\right) \bar{\psi}(\eta) d \eta \int \exp (-i y \cdot \eta) B_{N}(y) d y
$$

where $\bar{\psi}$ is a symbol of order zero such that $\bar{\psi}(\eta)=1$ on a conic neighborhood of support $q\left(I^{-1}(\eta)\right)$ and support $\bar{\psi}$ is in $\tilde{C}$.

$$
B_{N}(y)=\int_{-\infty}^{y_{1}} \exp \left((i \lambda+\varepsilon)\left(s-y_{1}\right)\right) H_{N}\left(s, y_{2}, \ldots, y_{n}\right) d s
$$

and

$$
H_{N}(y)=(2 \pi)^{-n / 2} \int \exp (i y \cdot I(\xi)) \psi(|\xi| / N) q(\xi) \hat{h}(\xi) d \xi .
$$

Let $M=(1 / i)\left|\left(I^{\prime}(\xi)\right)^{*} y-x\right|^{-2}\left(\left(I^{\prime}(\xi)\right)^{*} y-x\right) \cdot \partial / \partial \xi$ and choose $R^{\prime}$ so that $\left|\left(I^{\prime}(\xi)\right)^{*} y-x\right|>1$ for $|x| \leq R,|y|>R^{\prime}, \xi \in$ support $q$. Then we have for $|y|>R^{\prime}$ and any integer $r>0$

$$
H_{N}(y)=\left((2 \pi)^{-n} \int e^{i(y \cdot I(\xi)-x \cdot \xi)}\left(M^{*}\right)^{r}\left(\psi\left(\frac{|\xi|}{N}\right) q(\xi)\right) d \xi, h\right) .
$$

Letting $\varphi \in C_{0}^{\infty}\left(\mathbf{R}^{n}\right), \varphi(y)=1$ for $|y| \leq R^{\prime}$, (1.4) implies for all $m$

$$
\rho_{m}\left((1-\varphi) H_{N}(y)\right) \leq c_{m}\|h\|_{-1}
$$


where $\rho_{m}$ denotes the seminorm

$$
\rho_{m}(f)=\sup \sum_{|\alpha| \leq m}\left(1+|x|^{2}\right)^{m}\left|D^{\alpha} f\right|
$$

and $c_{m}$ is independent of $N$.

Since $\|f\|_{-s}=\sup \left\{(v, f) \mid v \in C_{0}^{\infty},\left\|(1-\Delta)^{s / 2} v\right\|_{0} \leq 1\right\}$, we have (assume $s$ is an even integer)

$$
\left\|\varphi H_{N}\right\|_{-s} \leq(2 \pi)^{-n}\left\|\left(\int e^{i(y \cdot I(\xi)-x \cdot \xi)} \frac{\varphi(y) \psi(|\xi| / N) q(\xi)}{\left(1+|I(\xi)|^{2}\right)^{s / 2}} d \xi, h\right)\right\|_{0} .
$$

As $I(\hat{\xi}) \neq 0$, we may assume that $C$ is chosen so that $I(\xi) \neq 0$ for $\xi \in C$ $\cap\{|\xi|=|\hat{\xi}|\}$. Then (1.6) implies for $S$ sufficiently large

$$
\left\|\varphi H_{N}\right\|_{-s} \leq c\|h\|_{-1} \text { and } c \text { is independent of } N \text {. }
$$

Choose $\rho \in C^{\infty}(\mathbf{R})$ such that $\rho(x)=1$ for $x<R^{\prime \prime}-1$ and $\rho(x)=0$ for $x>-R^{\prime \prime}$, where support $\varphi$ is contained in $y_{1}>-R^{\prime \prime}$. Then (1.3) and (1.5) imply for all $m$

$$
\rho_{m}\left(\rho\left(y_{1}\right) B_{N}(y)\right) \leq k_{m}\|h\|_{-1}
$$

where $k_{m}$ is independent of $N, \lambda$ and $\varepsilon$.

Using the definition of \|\|$_{-s}$ we have

$$
\begin{aligned}
\left\|\left(1+y_{1}^{2}\right)^{-1}\left(1-\rho\left(y_{1}\right)\right) B_{N}(y)\right\|_{-s} & \\
= & \sup _{\left\|(1-\Delta)^{s / 2}\right\|_{b} \leq 1} \int v(y)\left(1+y_{1}^{2}\right)^{-1} \\
& \cdot\left(1-\rho\left(y_{1}\right)\right) \int_{-\infty}^{y_{1}} e^{(i \lambda+e)\left(s-y_{1}\right)} H_{N}\left(s, y_{2}, \ldots, y_{n}\right) d s d y \\
= & \sup _{\left\|(1-\Delta)^{s / 2} v\right\|_{0} \leq 1} \int V(y) H_{N}(y) d y
\end{aligned}
$$

where

$$
V(y)=\int_{y_{1}}^{\infty}\left(1+s^{2}\right)^{-1}(1-\rho(s)) \exp \left((i \lambda+\varepsilon)\left(y_{1}-s\right)\right) v\left(s, y_{2}, \ldots, y_{n}\right) d s .
$$

Let $\psi \in C_{0}^{\infty}\left(\mathbf{R}^{n}\right)$ equal 1 on support $\varphi$. Since

$$
\left\|(1-\Delta)^{S / 2} \psi V\right\|_{0} \leq C\left\|(1-\Delta)^{S / 2} v\right\|_{0},
$$

where $C$ is independent of $\varepsilon$ and $\lambda$ for $0 \leq \varepsilon \leq 1$ and $|\lambda| \leq K$, (1.7) implies

$$
\begin{aligned}
& \sup _{\left\|(1-\Delta)^{s / 2} v\right\|_{b} \leq 1} \int V(y) H_{N}(y) d y \\
& \leq c C\|h\|_{-1}+\sup _{\left\|(1-\Delta)^{s / 2} v\right\|_{0} \leq 1} \int V(y)(1-\varphi(y)) H_{N}(y) d y .
\end{aligned}
$$


One also has $\sup _{y_{1} \in \mathbf{R}}\left\|V\left(y_{1}, \cdot\right)\right\|_{0} \leq C^{\prime}\|v\|_{0}$, and $\left\|(1-\Delta)^{S / 2} v\right\|_{0} \leq 1$ implies $\|v\|_{0}$ $\leq 1$. Thus an application of (1.5) (with $m=1$ ) yields

$$
\left\|\left(1+y_{1}^{2}\right)^{-1}\left(1-\rho\left(y_{1}\right)\right) B_{N}(y)\right\|_{-s} \leq k\|h\|_{-1}
$$

where $k$ is independent of $N, \lambda$ and $\varepsilon$.

Next we set

$$
M^{\prime}=(1 / i)\left|\left(\left(I^{-1}\right)^{\prime}(\eta)\right)^{*} x-y\right|^{-2}\left(\left(\left(I^{-1}\right)^{\prime}(\eta)\right)^{*} x-y\right) \cdot \partial / \partial \eta .
$$

Since $\left(\left(I^{-1}\right)^{\prime}(\eta)\right)^{*} x=\left(\left(\Lambda^{\prime}(0 \xi)\right)^{*}\right)^{-1} 0 x=\left(\left(\partial \tau \circ 0^{*} / \partial \xi_{1}^{\prime}(0 \xi)\right)^{-1}(0 x)_{1}, \ldots\right)$ where $\xi=I^{-1}(\eta)$, there is an $R_{0}>0$ such that $\left|\left(\left(I^{-1}\right)^{\prime}(\eta)\right)^{*} x-y\right|>1$ for $(0 x)_{1}$ $<-R_{0}, y_{1}>-R^{\prime \prime}-1, \eta \in \tilde{C}$. Hence for $(0 x)_{1}<-R_{0}$ and $r$ sufficiently large

$$
Q(D) u_{N}(x)
$$

$$
\begin{aligned}
= & \left((2 \pi)^{-n} \int \exp \left(i\left(x \cdot I^{-1}(\eta)-y \cdot \eta\right)\right)\left(M^{\prime}\right)^{* r}(\bar{\psi}(\eta)) d \eta,\left(1-\rho\left(y_{1}\right)\right) B_{N}\right) \\
& +(2 \pi)^{-n} \int \exp \left(i x \cdot I^{-1}(\eta)\right) \bar{\psi}(\eta) d \eta \int \exp (-i y \cdot \eta) \rho\left(y_{1}\right) B_{N}(y) d y .
\end{aligned}
$$

Now (1.8), (1.9) and (1.10) imply for all $m$

$$
\sup _{(0 x)_{1} \leq-R_{0}} \sum_{|\alpha| \leq m}\left|D^{\alpha} Q(D) u_{N}(x)\right| \leq K_{m}\|h\|_{-1}
$$

and since $Q(D) u_{N} \rightarrow Q(D) u$ in $L^{2}\left(\mathbf{R}^{n}\right)$ as $N \rightarrow \infty$ and $K_{m}$ is independent of $N$, we have

$$
\sup _{(0 x)_{1} \leq-R_{0}} \sum_{|\alpha| \leq m}\left|D^{\alpha} Q(D) u(x)\right|<K_{m}\|h\|_{-1} .
$$

To complete the proof we only need to observe

$$
(x(t), \xi(t))=(\hat{x}+(T-t) \partial \tau / \partial \xi(\hat{\xi}), \hat{\xi})
$$

for $t<-T$. Since $(0 \partial \tau / \partial \xi)_{1}=\left(\partial \tau \circ 0^{*}\right) / \partial \xi_{1}^{\prime}>0$, by taking $T_{0}$ sufficiently large we may assume $\left(0 x\left(-T_{0}\right)\right)_{1}<-R_{0}$. Since $\|h\|_{-1} \leq c\left(\|g\|_{-1}+\|u\|_{0}^{R}\right)$, the conclusion of Lemma 1.2 now follows from (1.11).

The following argument reduces the proof of Theorem 1.1 to the preceding lemma and the theorem of Hörmander cited earlier:

Let $(x(t), \xi(t))$ be the bicharacteristic of Lemma 1.2. Choose $R^{\prime}>R$ such that $\left\{x(t)|| t \mid \leq T_{0}\right\}$ is contained in $|x|<R^{\prime}-1$ and choose $f \in C_{0}^{\infty}\left(|x|<R^{\prime}\right)$ so that $f(x)=1$ for $|x| \leq R^{\prime}-1$. Let $\varphi(x, \xi)$ be a symbol of order zero with support in $\left\{V \cap(x, \xi)|| x \mid \leq R^{\prime}-1\right\}$ such that $\varphi(x, \xi)=1$ on a conic neighborhood of $\Gamma=\left\{(x(t), \xi(t))|| t \mid \leq T_{0}\right\}$. Note that $\Gamma \subset\{(x, \xi) \mid \operatorname{det} A(x, \xi)=0\}$. We 
call an operator $\varphi_{0}(x, D) u=\varphi(x, D) f u$ a cutoff to $\Gamma$. Given two cutoffs $\varphi_{0}$ and $\psi_{0}$ we say $\psi_{0}>\varphi_{0}$ if $\psi(x, \xi)=1$ on the support of $\varphi(x, \xi)$.

Given cutoffs, $\varphi_{0}$ and $\psi_{0}, \psi_{0}>\varphi_{0}$, let $P(x, D)$ be the operator with symbol $\varphi(x, \xi) P(x, \xi)$ and set $P_{0}(x, D)=P(x, D) f$. Similarly $\tau(x, D)$ is the operator with symbol $\psi(x, \xi) \tau(x, \xi)$ and $\tau_{0}(x, D)=\tau(x, D) f$. By construction, given $L u+$ $(i \lambda \pm \varepsilon) u=g$,

$$
\tau_{0} P_{0} u+M P_{0} u+(i \lambda \pm \varepsilon) P_{0} u=P_{0} g+M\left(P_{0}-I\right) u,
$$

where $M=P_{0} L-\tau_{0} P_{0}$ is an operator of order zero.

Let $\bar{\varphi}_{0}$ and $\bar{\psi}_{0}$ be cutoffs such that $\bar{\varphi}_{0}<\bar{\psi}_{0}<\varphi_{0}$. Then

$$
\begin{aligned}
L\left(\bar{\varphi}_{0}\left(P_{0}-I\right) u\right)= & -(i \lambda \pm \varepsilon) \bar{\varphi}_{0}\left(P_{0}-I\right) u+\left[L, \bar{\varphi}_{0}\left(P_{0}-I\right)\right] \bar{\psi}_{0} u \\
& +\left[L, \bar{\varphi}_{0}\left(P_{0}-I\right)\right]\left(I-\bar{\psi}_{0}\right) u+\bar{\varphi}_{0}\left(P_{0}-I\right) g .
\end{aligned}
$$

Let $\Lambda^{s}$ denote the operator with symbol $|\xi|^{s} \psi(x, \xi)$ and $\Lambda_{0}^{s}=\Lambda^{s}(x, D) f$. Since the symbol of $I-P_{0}$ is the orthogonal projection onto $N(x, \xi)^{\perp}$ on the support of $\bar{\varphi}_{0}$, $\left\|\Lambda_{0}^{1} P_{0}\left(\bar{\varphi}_{0}\left(P_{0}-I\right)\right) u\right\|_{s} \leq C_{s}\left(\left\|\bar{\psi}_{0} u\right\|_{s}+\|u\|_{0}^{R^{\prime}}\right)$. However, since $A(x, \xi)$ is invertible on $N(x, \xi)^{\perp}$ for $(x, \xi) \in U$, for $(x, \xi) \in U,\left(\Lambda_{0}^{s} L\right)^{*}\left(\Lambda_{0}^{s} L\right)+\left(\Lambda_{0}^{s+1} P_{0}\right)^{*}\left(\Lambda_{0}^{s+1} P_{0}\right)$ is an elliptic operator on the support of $\varphi(x, \xi) f(x)$. Thus by Gårding's inequality

$$
\begin{aligned}
\left\|\bar{\varphi}_{0}\left(P_{0}-I\right) u\right\|_{s+1}^{2} \leq & C\left(\bar{\varphi}_{0}\left(P_{0}-I\right) u,\left(\Lambda_{0}^{s} L\right)^{*}\left(\Lambda_{0}^{s} L\right) \bar{\varphi}_{0}\left(P_{0}-I\right) u\right) \\
& +C\left(\bar{\varphi}_{0}\left(P_{0}-I\right) u,\left(\Lambda_{0}^{s+1} P_{0}\right)^{*}\left(\Lambda_{0}^{s+1} P_{0}\right) \bar{\varphi}_{0}\left(P_{0}-I\right) u\right)+C\left(\|u\|_{0}^{R^{\prime}}\right)^{2} .
\end{aligned}
$$

Hence

(1.14) $\left\|\bar{\varphi}_{0}\left(I-P_{0}\right) u\right\|_{s+1} \leq c_{s}\left(\left\|L\left(\bar{\varphi}_{0}\left(I-P_{0}\right) u\right)\right\|_{s}+\left\|\Lambda_{0}^{1} P_{0}\left(\bar{\varphi}_{0}\left(I-P_{0}\right) u\right)\right\|_{s}+\|u\|_{0}^{R^{\prime}}\right)$.

Hence, combining (1.13) and (1.14) we have

$$
\left\|\bar{\varphi}_{0}\left(P_{0}-I\right) u\right\|_{s+1} \leq K_{s}\left(\left\|\bar{\psi}_{0} u\right\|_{s}+\|u\|_{0}^{R^{\prime}}+\|g\|_{s}\right)
$$

where $K_{s}$ is independent of $\varepsilon$ and $\lambda$ for $0<\varepsilon \leq 1,|\lambda|<K$. Since $\left[\bar{\varphi}_{0}, M\right]$ is order -1 , if we rewrite $(1.12)$ as $\left(\tau_{0}+M+(i \lambda \pm \varepsilon) I\right) P_{0} u=p$, (1.15) implies

$$
\left\|\bar{\varphi}_{0} p\right\|_{s+1} \leq c_{s}\left(\left\|\bar{\psi}_{0} u\right\|_{s}+\|u\|_{0}^{R^{\prime}}+\|g\|_{s+1}\right) \text {. }
$$

By Lemma 1.2

$$
\left\|\varphi_{ \pm} u\right\|_{s+1} \leq K_{s+1}\left(\|g\|_{0}+\|u\|_{0}^{R^{\prime}}\right)
$$

and the symbols $\varphi_{ \pm}(x, \xi)=P(\xi)$ on $N_{ \pm}$. Hence

$$
\left\|\rho_{ \pm} P_{0} u\right\|_{s+1} \leq K_{s+1}\left(\|g\|_{0}+\|u\|_{0}^{R^{\prime}}\right)
$$

for suitably chosen $\rho_{ \pm}$, pseudo-differential operators of order zero with $\rho_{ \pm}(x, \xi)$ $=1$ on conic neighborhoods of $(x( \pm T), \xi( \pm T))$. Setting $s=0$ in (1.16) and 
(1.17), it now follows from Proposition 3.5.1 of [5] (see (1.1)) that there is a cutoff $\rho_{0}, \rho_{0}<\bar{\varphi}_{0}$, such that

$$
\left\|\rho_{0} P_{0} u\right\|_{1} \leq c\left(\|g\|_{1}+\|u\|_{0}^{R^{\prime}}\right)
$$

In Proposition 3.5.1 Hörmander considers scalar operators. However, since the top order part of our operator, $\tau_{0}$, is scalar, the proof in [5] goes through unchanged.

Combining (1.15) and (1.18),

$$
\left\|\rho_{0} u\right\|_{1} \leq c_{1}\left(\|g\|_{1}+\|u\|_{0}^{R^{\prime}}\right)
$$

Choosing new cutoffs which we again call $\bar{\varphi}_{0}$ and $\bar{\psi}_{0}$ such that $\bar{\varphi}_{0}<\bar{\psi}_{0}<\rho_{0}$, (1.15) now implies

$$
\left\|\bar{\varphi}_{0}\left(P_{0}-I\right)\right\|_{2} \leq K\left(\|g\|_{1}+\|u\|_{0}^{R^{\prime}}\right) \text { and }\left\|\bar{\varphi}_{0} p\right\|_{2} \leq K\left(\|g\|_{2}+\|u\|_{0}^{R^{\prime}}\right) .
$$

Using (1.17) with $s=1$ and applying Hörmander's theorem again, we have a cutoff $\rho_{1}<\bar{\varphi}_{0}$ such that $\left\|\rho_{1} u\right\|_{2} \leq c\left(\|g\|_{2}+\|u\|_{0}^{R^{\prime}}\right)$. Repeating this argument, we conclude given $s$ there is cutoff $\rho_{s-1}$ such that

$$
\left\|\rho_{s-1} u\right\|_{s} \leq C_{s}\left(\|g\|_{s}+\|u\|_{0}^{R^{\prime}}\right)
$$

where $C_{s}$ is independent of $\varepsilon$ and $\lambda$ for $0<\varepsilon \leq 1$. In particular $\rho_{s-1}(x, \xi)=1$ on a conic neighborhood of the original point $\left(x_{0}, \xi_{0}\right)$ (see remarks preceding Lemma 1.2). Since $\left(x_{0}, \xi_{0}\right)$ was an arbitrary point in $S_{R}$ and $S_{R}$ is compact, we can find a finite set of points $\left(x_{0}^{i}, \xi_{0}^{i}\right) \in S_{R}, i=1, \ldots, N$, such that $\sum_{i=1}^{N}\left(\rho_{s-1}^{i}(x, \xi)\right)^{2} \geq 1$ on a conic neighborhood of $S_{R}$. Since $L$ is elliptic off any conic neighborhood of $S$, there is an $\alpha \in C_{0}^{\infty}\left(\mathbf{R}^{n}\right), \alpha=1$ for $|x| \leq R$, such that

$$
\|\alpha u\|_{s} \leq C_{s}\left(\|(L+i \lambda+\varepsilon) u\|_{s-1}+\sum_{i=1}^{N}\left\|\rho_{s-1}^{i}(u)\right\|_{s}+\|u\|_{0}^{R^{\prime}}\right) .
$$

Thus, combining (1.19) and (1.20), we have Theorem 1.1.

The hypothesis $\varepsilon>0$ was only used in Lemma 1.2. Hence the proof of Theorem 1.1 also yields

Theorem 1.3. Let $L u+i \lambda u=g, g \in C_{0}^{\infty}(|x|<R), u \in L^{2}(\mathbf{R}), u=0$ for $|x|>R$. Then given $s$ there is $a C_{s}$ independent of $\lambda$ and $u$ such that

$$
\|\alpha u\|_{s} \leq C_{s}\left(\|g\|_{s}+\|u\|_{0}\right)
$$

Theorem 1.3 enables us to prove the point spectrum of $L$ is discrete (cf. [7, Theorem 3.1] and [10]).

Corollary 1.4. The eigenvalues of $L$ have no accumulation points and the nonzero eigenvalues have finite multiplicity. 
Proof. Since $i L$ is selfadjoint, the eigenvalues of $L$ are purely imaginary. Consider $f \in L^{2}\left(\mathbf{R}^{n}\right)$ with $(L+i \lambda) f=0, \lambda \neq 0$. Let $J_{\varepsilon}$ be the standard "mollification" operator. Then $J_{e} f \in L^{2}\left(\mathbf{R}^{n}\right) \cap C^{\infty}$ and

$$
\left(L_{0}+i \lambda\right) J_{\varepsilon} f=\left(L_{0}-L\right) J_{\varepsilon} f+\left[L, J_{\varepsilon}\right] f \text {. }
$$

Since $\left[L, J_{\varepsilon}\right]$ is a bounded operator on $L^{2}\left(\mathbf{R}^{n}\right)$ and $\left[L, J_{\varepsilon}\right] v=0$ for all $v$ with support in $|x|>R+\varepsilon, J_{\varepsilon} f$ satisfies the hypotheses of Theorem 3.3 of [7]. This theorem is proved via the Radon transform and requires $n$ odd. Hence $J_{e} f=0$ for $|x|>R+\varepsilon$. Since $J_{\varepsilon} f \rightarrow f$ as $\varepsilon \rightarrow 0$, we have $f=0$ for $|x|>R$.

Suppose $L$ has an infinite sequence of orthonormal eigenfactors $f_{n}$ with eigenvalues $i \lambda_{n}$ and $\lambda_{n} \rightarrow \lambda$ where $\lambda_{n} \neq 0$ though $\lambda$ may be. Then, by Theorem $1.1,\left\|f_{n}\right\|_{1} \leq C$ where $C$ is independent of $n$. Since $\left\{f\left|\|f\|_{1} \leq C, f=0,\right| x \mid>R\right\}$ is precompact in $L^{2}$, we have a contradiction which proves the corollary.

2. Construction of generalized eigenfunctions. In this section we will construct solutions to $L u+i \lambda u=0, \lambda \in \mathbf{R}$, by the limiting absorption method. These eigenfunctions will satisfy "radiation" and mild regularity conditions which will enable us to prove a strong decay result (Theorem 2.2) for two subspaces of initial data. In $\$ 3$ we will show the sum of these subspaces is dense in $L^{2}\left(\mathbf{R}^{n}\right) \ominus H_{0}$.

Let $\tau_{j}(\omega)$ and $r_{j}(\omega), j=1, \ldots, k$, be functions on the unit sphere $S^{n-1}$ such that $\left\|r_{j}(\omega)\right\|=1, \tau_{1} \geq \tau_{2} \geq \cdots \geq \tau_{k}$, and for $A_{0}(\xi)=\sum_{j=1}^{n} A_{j}^{0} \xi_{j}$ we have

$$
A_{0}(\omega) r_{j}(\omega)=\tau_{j}(\omega) r_{j}(\omega) \quad \forall \omega \in S^{n-1} .
$$

The functions $\tau_{j}(\omega)$ and $r_{j}(\omega)$ may be chosen measurable on $S^{n-1}$.

Since $\partial \tau / \partial \xi(x, \xi) \neq 0$ for $(x, \xi)$ in a neighborhood of $S$ and $\xi \cdot \partial \tau / \partial \xi(x, \xi)=0$ on $S$, the set $S_{0}=\left\{\omega \in S^{n-1} \mid \operatorname{det} A_{0}(\omega)=0\right\}$ is a smooth submanifold of $S^{n-1}$ of codimension 1 with a finite number of components. Hence $S_{0}$ is a closed subset of measure zero in $S^{n-1}$.

For $\xi \in \mathbf{R}^{n}-\{0\}$ let $\varphi_{j}(x, \xi)=e^{i x \cdot \xi} r_{j}(\xi /|\xi|)$. Then

$$
\left(L-i \tau_{j}(\xi)\right) \varphi_{j}(x, \xi)=\left(L-L_{0}\right) \varphi_{j}(x, \xi) \equiv g_{j}(x, \xi)
$$

is $C^{\infty}$ in $x$ and has support in $x$ contained in $|x|<R$. Let $v_{j, \varepsilon}^{+}(x, \xi)$ and $v_{j, e}^{-}(x, \xi)$ be the square-integrable solutions to

$$
\left(L-\left(i \tau_{j}(\xi) \pm \varepsilon\right)\right) v_{j, \varepsilon}^{ \pm}=-g_{j},
$$

and let \|\|$_{s}^{R}$ denote the Sobolev $s$-norm ( $s$ a positive integer) over the ball $|x| \leq R$.

By Theorem 1.1 for $\xi$ in a compact set $C$ in $\mathbf{R}^{n}-\{0\}$ and $0<\varepsilon \leq 1$

$$
\left\|\nu_{j, \varepsilon}^{ \pm}(\cdot, \xi)\right\|_{s}^{R} \leq C_{s}\left(K_{s}+\left\|v_{j, \varepsilon}^{ \pm}(\cdot, \xi)\right\|_{0}^{R^{\prime}}\right)
$$


where $C_{s}$ is independent of $\xi$ and $\varepsilon$ and $K_{s}=\sup _{\xi \in C}\left\|g_{j}(\cdot, \xi)\right\|_{s}<\infty$. We set $M=\left\{\xi \in \mathbf{R}^{n}-\{0\}|\xi /| \xi \mid \notin S_{0}, \operatorname{det}\left(A_{0}(\xi)-\lambda I\right) \neq 0\right.$ for $i \lambda$ in point spectrum $L\}$.

By Corollary 1.4 the point spectrum of $L$ has no accumulation points. Hence $M$ is an open subset of $\mathbf{R}^{n}$ and $M^{c}$ has measure zero.

Lemma 2.1. Let $C$ be compact subset of $M$. Then

$$
\sup _{1 \geq \varepsilon>0 ; \xi \in C}\left\|v_{j, \varepsilon}^{+}(\cdot, \xi)\right\|_{0}^{R^{\prime}}<\infty \text { and } \sup _{1 \geq \varepsilon>0 ; \xi \in C}\left\|v_{j, \varepsilon}^{-}(\cdot, \xi)\right\|_{0}^{R^{\prime}}<\infty .
$$

Proof. We will give the proof for $v_{j, \varepsilon^{-}}^{+}$The proof for $v_{j, \varepsilon}^{-}$is identical. Assume $\sup _{1 \geq e>0 ; \xi \in C}\left\|v_{j, \varepsilon}^{+}(\cdot, \xi)\right\|_{0}^{R^{\prime}}=\infty$, and set $u_{\varepsilon}=v_{j, e}^{+} /\left\|v_{j, e}^{+}\right\|_{0}^{R^{\prime}}$. Then by Rellich's compactness criterion and a Cantor diagonal argument we conclude from (2.1): there are sequences $\varepsilon_{i} \rightarrow 0, \xi_{i} \rightarrow \xi_{0}$, and $u \in C^{\infty}(|x|<R)$ such that $\tau_{j}\left(\xi_{i}\right) \rightarrow \lambda \neq 0$, $\left\|v_{j, \varepsilon_{i}}^{+}\left(\cdot, \xi_{i}\right)\right\|_{0}^{R^{\prime}} \nearrow \infty$, and

$$
\left\|u_{\varepsilon_{i}}\left(x, \xi_{i}\right)-u\right\|_{s}^{R} \rightarrow 0 \text { as } i \rightarrow \infty \text {, for all } s .
$$

Hence setting $h_{i}=\left(L_{0}-\left(i \tau_{j}\left(\xi_{i}\right)+\varepsilon_{i}\right)\right) u_{\varepsilon_{i}}$ we have

$$
\left\|h_{i}-\left(L_{0}-L\right) u\right\|_{s} \rightarrow 0
$$

for all $s$, and $h_{i}=0$ for $|x|>R$.

To study the convergence of $u_{\varepsilon_{i}}$ for $|x|>R$ we use the Radon transform: for $f \in C_{0}^{\infty}\left(\mathbf{R}^{n}\right)$ define

$$
R f=\frac{1}{2}\left(\frac{-\partial}{\partial s}\right)^{(n-1) / 2}\left(\int_{x \cdot \omega=s} f d A\right)
$$

The mapping $R$ extends to a unitary map of $L^{2}\left(\mathbf{R}^{n}\right)$ onto the even or odd functions in $L^{2}\left(R \times S^{n-1}\right)$ as $(n-1) / 2$ is even or odd (cf. [7, Theorem 2.2]). Interpreting $\partial / \partial s$ in the sense of distributions, one computes

$$
\left(A_{0}(\omega) \partial / \partial s-\left(i \tau_{j}\left(\xi_{i}\right)+\varepsilon_{i}\right)\right) R u_{\varepsilon_{i}}=R h_{i}
$$

where $R h_{i}(s, \omega)=0,|s| \geq R$. For $\omega \in S^{n-1}-S_{0}$ we choose $k(\omega)$ so that $\tau_{1}(\omega) \geq \cdots \geq \tau_{k(\omega)}(\omega)>0>\tau_{k(\omega)+1}(\omega) \geq \cdots \geq \tau_{k}(\omega)$. Then, setting $\lambda_{i}=\tau_{j}\left(\xi_{i}\right)$, (2.3) implies

$$
\begin{aligned}
R u_{\varepsilon_{i}}(s, \omega)= & \sum_{l=1}^{k(\omega)}-\int_{s}^{\infty}\left(\exp \left(\frac{i \lambda_{i}+\varepsilon_{i}}{\tau_{l}(\omega)}\right)(s-\sigma)\right) \frac{\overline{r_{l}(\omega)} \cdot R h_{i}(\sigma, \omega)}{\tau_{l}(\omega)} d \sigma r_{l}(\omega) \\
& +\sum_{l=k(\omega)+1}^{k} \int_{-\infty}^{s}\left(\exp \left(\frac{i \lambda_{i}+\varepsilon_{i}}{\tau_{l}(\omega)}\right)(s-\sigma)\right) \frac{\overline{r_{l}(\omega)} \cdot R h_{i}(\sigma, \omega)}{\tau_{l}(\omega)} d \sigma r_{l}(\omega)
\end{aligned}
$$

for $\omega \in S^{n-1}-S_{0}$. 
By (2.2) we have

$$
\lim _{i \rightarrow \infty} \operatorname{Re} \int_{\mathbf{R}^{n}} \overline{u_{\varepsilon_{i}}} \cdot h_{i}=\lim _{i \rightarrow \infty} \operatorname{Re} \int_{\mathbf{R}^{n}} \overline{u_{\varepsilon_{i}}} \cdot\left(L_{0}-L\right) u_{\varepsilon_{i}}=0 .
$$

Hence since $R$ is an isometry

$$
0=\lim _{i \rightarrow \infty} \operatorname{Re} \int_{\mathbf{R} \times S^{n-1}} \overline{R u_{\varepsilon_{i}}} \cdot R h_{i} d \omega d \sigma .
$$

Substituting (2.4) into (2.5), one obtains after brief manipulation

$$
\begin{aligned}
0= & \lim _{i \rightarrow \infty} \int_{S^{n-1}} \sum_{l=1}^{k} \frac{-1}{\left|\tau_{l}(\omega)\right|} \int_{-\infty}^{\infty} d s \int_{-\infty}^{\infty} d \sigma \\
& \cdot \exp \left(-\varepsilon_{i}|s-\sigma| /\left|\tau_{l}(\omega)\right|\right) \overline{\left(\exp \left(-i \lambda_{i} s / \tau_{l}(\omega)\right) R h_{i}(s, \omega) \cdot \overline{r_{l}(\omega)}\right)} \\
& \cdot\left(\exp \left(-i \lambda_{i} \sigma / \tau_{l}(\omega)\right) R h_{i}(\sigma, \omega) \cdot \overline{r_{l}(\omega)}\right) .
\end{aligned}
$$

Since $\exp \left(\left(-\varepsilon_{i} /\left|\tau_{l}(\omega)\right|\right)|s-\sigma|\right)$ is a positive kernel and $\left\{R h_{i}(\cdot, \omega)\right\}$ is converging in $L^{2}(d s)$ for all $\omega$ by (2.2), it follows by Fatou's lemma

$$
0=\sum_{l=1}^{k} \frac{-1}{\left|\tau_{l}(\omega)\right|}\left|\int_{-\infty}^{\infty} \exp \left(-i \lambda s / \tau_{l}(\omega)\right) R h(s, \omega) \cdot \overline{r_{l}(\omega)} d s\right|^{2}
$$

a.e. in $\omega$, where $h=\left(L_{0}-L\right) u$. Hence

$$
0=\int_{-\infty}^{\infty} \exp \left(i \lambda s / \tau_{l}(\omega)\right) R h(s, \omega) \cdot r_{l}(\omega) d s
$$

a.e. in $\omega, l=1, \ldots, k$.

Integrating by parts in (2.4) we have

$$
\begin{aligned}
R u_{\varepsilon_{i}}(s, \omega)=\sum_{l=1}^{k(\omega)}-\left[\frac{\overline{r_{l}(\omega)} \cdot R h_{i}(s, \omega) r_{r}(\omega)}{i \lambda_{i}+\varepsilon_{i}}\right. & \\
& \left.\quad+\int_{s}^{\infty}\left(\exp \left(\frac{i \lambda_{i}+\varepsilon_{i}}{\tau_{l}(\omega)}\right)(s-\sigma)\right) \frac{\overline{r_{l}(\omega)} \cdot \partial R h_{i} / \partial \sigma}{i \lambda_{i}+\varepsilon_{i}} d \sigma r_{l}(\omega)\right] \\
+ & \sum_{k(\omega)+1}^{k}\left[\frac{-\overline{r_{l}(\omega)} \cdot R h_{i}(s, \omega) r_{l}(\omega)}{i \lambda_{i}+\varepsilon_{i}}\right. \\
& \left.+\int_{-\infty}^{s}\left(\exp \left(\frac{i \lambda_{i}+\varepsilon_{i}}{\tau_{l}(\omega)}\right)(s-\sigma)\right) \frac{\overline{r_{l}(\omega)} \cdot \partial R h_{i} / \partial \sigma}{i \lambda_{i}+\varepsilon_{i}} d \sigma r_{l}(\omega)\right] .
\end{aligned}
$$

Since (2.2) implies $R h_{i}$ converges uniformly together with its derivatives to $R h$ and $\lambda_{i}$ is bounded away from zero, it follows from (2.7) that $\left|R u_{\varepsilon_{i}}(s, \omega)\right|<c \forall i$, $s \in \mathbf{R}, \omega \in S^{n-1}-S_{0}$. Moreover, $R u_{\varepsilon_{i}}(s, \omega)$ converges pointwise on $\mathbf{R} \times\left\{S^{n-1}\right.$ $\left.-S_{0}\right\}$ to 


$$
\begin{aligned}
v(s, \omega)= & \sum_{l=1}^{k(\omega)}-\int_{s}^{\infty} \exp \left(\frac{i \lambda}{\tau_{l}(\omega)}(s-\sigma)\right) \frac{\overline{r_{l}(\omega)} \cdot R h(\sigma, \omega)}{\tau_{l}(\omega)} d \sigma r_{l}(\omega) \\
& +\sum_{l=k(\omega)+1}^{k} \int_{-\infty}^{s} \exp \left(\frac{i \lambda}{\tau_{l}(\omega)}(s-\sigma)\right) \frac{\overline{r_{l}(\omega)} \cdot R h(\sigma, \omega)}{\tau_{l}(\omega)} d \sigma r_{l}(\omega) .
\end{aligned}
$$

By (2.6), $v(s, \omega) \in L^{2}\left(\mathbf{R} \times S^{n-1}\right)$. Making use of (a) $R$ is an isometry, and (b) $R$ maps $C_{0}^{\infty}\left(\mathbf{R}^{n}\right)$ to $C_{0}^{\infty}\left(\mathbf{R} \times S^{n-1}\right)$, we conclude from the preceding that $u_{\varepsilon_{i}}$ converges in the sense of distributions to an extension $\tilde{u}$ of $u$ with $R \tilde{u}=v$, and in the sense of distributions $\left(L_{0}-i \lambda\right) \tilde{u}=\left(L_{0}-L\right) \tilde{u}$. Moreover, by the Lebesgue dominated convergence theorem $R u_{\varepsilon_{i}}$ converges to $R \tilde{u}$ in $L^{2}\left(\left\{|s|<R^{\prime}\right\} \times S^{n-1}\right)$ and thus (see (2.12)) $u_{e_{i}}$ converges to $\tilde{u}$ in $L^{2}\left(|x|<R^{\prime}\right)$. Thus $\tilde{u} \not \equiv 0$ and $i \lambda$ is an eigenvalue of $L$. This contradicts $C \subset \subset M$ and completes the proof.

Given a set $C \subset \subset M$, Lemma 2.1 and the estimate (2.1) imply for all $s$

$$
\sup _{\xi \in C ; 0<\varepsilon \leq 1}\left\|v_{j, \varepsilon}^{ \pm}(\cdot, \xi)\right\|_{s}^{R}<\infty
$$

Hence $\left\{v_{j, \varepsilon}^{ \pm}(x, \xi) \mid 0<\varepsilon \leq 1\right\}$ is a weakly compact set in $L^{2}(\{|x|<R\} \times C)$ for all $C \subset \subset M$. Choosing $C_{m} \nearrow M, C_{m} \subset \subset M$, and using a Cantor diagonal argument, we can choose $\varepsilon_{i} \rightarrow 0$ so that $v_{j, \varepsilon_{i}}^{ \pm}$converges weakly to a function $v_{j}^{ \pm}$on $\{|x|<R\} \times M$ in $L^{2}\left(\{|x|<R\} \times C_{m}\right)$ for all $m$. Since the weak closure of a set is contained within its closed convex hull $v_{j}^{ \pm}$satisfies (2.9) with $s=0$. [It is possible to choose $v_{j}^{ \pm}$so that it satisfies (2.9) for all $s$, but we will not use this.]

From this point on we will consider only $v_{j}^{+}$but our arguments will apply equally well to $v_{j}^{-}$. The formula (2.7) remains valid with $u_{\varepsilon_{i}}=v_{j, \varepsilon_{i}}^{+}, \lambda_{i}=\tau_{j}(\xi)$, and $h_{i}=\left(L_{0}-L\right) v_{j, \varepsilon_{i}}^{+}-g_{j}(x, \xi)$ and we conclude

$$
\left|R v_{j, \varepsilon_{i}}^{+}(s, \omega, \xi)\right|<K_{m}, \quad s \in \mathbf{R}, \omega \in S^{n-1}-S_{0}
$$

and $\xi \in C_{m}$. Thus, again using weak compactness and a Cantor diagonal argument, we conclude there is a subsequence of the $R v_{j, e_{i}}^{+}$which converges weakly to a function $R v_{j}^{+}$on $\mathbf{R} \times S^{n-1} \times M$ in $L^{2}\left(\{|s|<m\} \times S^{n-1} \times C_{m}\right)$ for all $m$. We denote this subsequence again by $R v_{j, e_{i}}^{+}$. Since the weak closure of a set is contained in its closed convex hull, we have $\left|R v_{j}^{+}(s, \omega, \xi)\right| \leq K_{m}$ a.e. for $(s, \omega, \xi) \in \mathbf{R} \times S^{n-1} \times C_{m}$, and

$$
\begin{aligned}
& R v_{j}^{+}(s, \omega, \xi) \cdot \overline{r(\omega)}=0, \text { for } s>R, l=1, \ldots, k(\omega) \\
& \text { and for } s<-R, l=k(\omega)+1, \ldots, k \text {, for }(s, \omega, \xi) \in \mathbf{R} \times S^{n-1} \times M
\end{aligned}
$$

The notation $R v_{j}^{+}$is justified as follows. For $\varphi \in C_{0}^{\infty}\left(\mathbf{R}^{n}\right)$ the inverse of $R$ is given by

$$
\varphi(x)=\frac{1}{2} \int_{S^{n-1}}\left(-\frac{\partial}{\partial s}\right)^{(n-1) / 2} R \varphi(\omega \cdot x, \omega) d \omega
$$


cf. [7, Lemma 2.1]. Hence the square-integrable functions on $\mathbf{R}^{n}$ given by

$$
u^{n}(x, \xi)=R^{-1}\left\{R v_{j}^{+} \uparrow|s|<n\right\},
$$

$n=1, \ldots, \infty$, satisfy $u^{n}(x, \xi)=u^{n+1}(x, \xi)$ for $|x|<n$. Defining $u(x, \xi)$ $=u^{n}(x, \xi)$ for $|x| \leq n$, we have $u(x, \xi) \in L_{\text {loc }}^{2}\left(\mathbf{R}^{n} \times M\right)$. Given $\psi(\xi) \in C_{0}^{\infty}(M)$ and $\varphi \in C_{0}^{\infty}\left(\mathbf{R}^{n}\right)$, we have

$$
\begin{aligned}
\int_{\mathbf{R}^{n} \times M} \psi(\xi) \overline{\varphi(x)} u(x, \xi) & =\int_{\mathbf{R} \times S^{n-1} \times M} \psi(\xi) \overline{R \varphi(s, \omega)} \cdot R v_{j}^{+}(s, \omega, \xi) \\
& =\lim _{i \rightarrow \infty} \int_{\mathbf{R} \times S^{n-1} \times M} \psi(\xi) \overline{R \varphi(s, \omega)} \cdot R v_{j, e_{i}}^{+}(s, \omega, \xi) \\
& =\lim _{i \rightarrow \infty} \int_{\mathbf{R}^{n} \times M} \psi(\xi) \varphi(x) v_{j, e_{i}}^{+}(x) .
\end{aligned}
$$

Thus $v_{j}^{+}(x, \xi)=u(x, \xi)$ a.e. for $|x|<R$ and we define $v_{j}^{+}(x, \xi)=u(x, \xi)$ a.e. for $|x|>R$.

Let $V=\left\{f \in L^{2}\left(\mathbf{R}^{n}\right) \mid R f\right.$ has compact support $\}$, and set $r_{j}(\xi)=r_{j}(\xi /|\xi|)$, $\tau_{j}(\xi)=|\xi| \tau_{j}(\xi /|\xi|)$. We define an "outgoing representation" $\mathscr{F}_{+} f$ for $f \in V$ by

$$
\begin{aligned}
\mathscr{F}_{+} f & =\tilde{f}_{+}(\xi)=\hat{f}(\xi)+(2 \pi)^{-n / 2} \sum_{j=1}^{k} r_{j}(\xi) \int_{\mathbf{R} \times S^{n-1}} R f(s, \omega) \cdot \overline{R v_{j}^{+}(s, \omega, \xi)} \\
& \equiv \sum_{j=1}^{k} r_{j}(\xi) \tilde{f}_{j, 0}^{+}(\xi) .
\end{aligned}
$$

The "incoming representation" $\mathscr{F}_{-}$is defined analogously substituting $v_{j}^{-}$for $v_{j}^{+}$. We will eventually show $\mathscr{F}_{+}$extends to an isometry from $L^{2}\left(\mathbf{R}^{n}\right) \ominus H_{0}$ onto $L^{2}\left(\mathbf{R}^{n}\right)$. The next theorem is the first step in this direction. Following Lax and Phillips we define "incoming" and "outgoing" subspaces $D^{-}=\left\{f \in L^{2}\left(\mathbf{R}^{n}\right) \mid\right.$ $R f(s, \omega) \cdot \overline{r_{j}(\omega)}=0$ for $s<R, j=1, \ldots, k(\omega) ; R f(s, \omega) \cdot \overline{r_{j}(\omega)}=0$ for $s$ . $>-R, j=k(\omega)+1, \ldots, k\}, D^{+}=\left\{f \in L^{2}\left(\mathbf{R}^{n}\right) \mid R f(s, \omega) \cdot r_{j}(\omega)=0\right.$ for $s$ $\left.>-R, j=1, \ldots, k(\omega), R f(s, \omega) \cdot \overline{r_{j}(\omega)}=0, s<R, j=k(\omega)+1, \ldots, k\right\}$ respectively.

Theorem 2.1. $\mathscr{F}_{ \pm}$extends to an isometry from $H_{\mp}=\left\{\overline{U(t) f \mid f \in D_{\mp}, t \in \mathbf{R}}\right\}$ onto $L^{2}\left(\mathbf{R}^{n}\right)$.

Proof. This proof follows [9, Theorem 2].

We give the proof for $\mathscr{F}_{+}$; the proof for $\mathscr{F}_{-}$is identical.

Since $R\left(U_{0}(t) f\right)(s, \omega)=\sum_{j=1}^{k}\left(R f\left(s-\tau_{j}(\omega) t, \omega\right) \cdot \overline{r_{j}(\omega)}\right) r_{j}(\omega), U_{0}(t)$ maps $V$ onto $V$. $U(t)$ maps functions of compact support to functions of compact support. Letting $c_{0}=\max _{|\omega|=1}\left\{\left|\tau_{j}(\omega)\right| \mid j=1, \ldots, k\right\}$, the standard domain of dependence argument shows $U(t) f=U_{0}(t) f$, if $f=0$ for $|x|<R+c_{0} t$. Thus $U(t)$ maps $V$ onto $V$ for all $t$. 
Given $\varphi \in C_{0}^{\infty}\left(\mathbf{R}^{n}\right)$ set

$$
\tilde{\varphi}_{j, \varepsilon}^{+}(t, \xi)=\int_{\mathbf{R}^{n}} U(t) \varphi \cdot \overline{\left[e^{i x \cdot \xi} r_{j}(\xi)+v_{j, \varepsilon}^{+}(x, \xi)\right]} d x .
$$

Since $L\left(e^{i x \cdot \xi} r_{j}+v_{j, \varepsilon}^{+}\right)=i \tau_{j}(\xi)\left(e^{i x \cdot \xi} r_{j}+v_{j, \varepsilon}^{+}\right)+\varepsilon v_{j, \varepsilon}^{+}$in the sense of distributions, one has

$$
\frac{\partial \tilde{\varphi}_{j, \varepsilon}^{+}}{\partial t}=i \tau_{j}(\xi) \varphi_{j, \varepsilon}^{+}-\varepsilon \int U(t) \varphi \cdot \overline{v_{j, \varepsilon}^{+}(x, \xi)} d x
$$

Thus

$$
\tilde{\varphi}_{j, \varepsilon}^{+}(t, \xi)=\exp \left(i \tau_{j}(\xi) t\right) \tilde{\varphi}_{j, \varepsilon}^{+}(0, \xi)+\varepsilon \int_{0}^{t} \exp \left(i \tau_{j}(\xi)\left(t-t^{\prime}\right)\right) m\left(t^{\prime}\right) d t^{\prime}
$$

where $m(t)=-\int U(t) \varphi \cdot \overline{v_{j, e}^{+}(x, \xi)} d x$. Since the Fourier transform is unitary, we can replace $\varphi$ in (2.15) by an arbitrary $f \in V$. Then we have for $\psi \in C_{0}^{\infty}(M)$

$$
\begin{aligned}
& \int \psi(\xi) \tilde{f}_{j, \varepsilon_{i}}^{+}(t, \xi) d \xi \\
& =\int \psi(\xi) \exp \left(i \tau_{j}(\xi) t\right) \tilde{f}_{j, \varepsilon_{i}}^{+}(0, \xi) d \xi \\
& \quad+\varepsilon_{i} \int_{0}^{t} d t^{\prime} \int_{\mathbf{R} \times S^{n-1} \times M} \exp \left(i \tau_{j}(\xi)\left(t-t^{\prime}\right)\right) \psi(\xi) R U(t) f \cdot \overline{R v_{j, \varepsilon_{i}}^{+}} d \xi
\end{aligned}
$$

Note that the proof showing $U(t)$ maps $V$ to $V$ implies given $f \in V$ and $[-T, T]$ there is a $\rho$ such that $R(U(t) f)(s, \omega)=0$ for $|s|>\rho$ and $t \in[-T, T]$. Thus, using the estimate (2.10), we see that the last integral in (2.16) tends to zero as $i \rightarrow \infty$. Since $R v_{j, \varepsilon_{i}}^{+}$converges weakly to $R v_{j}^{+}$in $L^{2}\left(\{|s|<m\} \times S^{n-1} \times C_{m}\right)$ for all $m$, we have for all $\psi \in C_{0}^{\infty}(M)$

$$
\int_{M} \psi(\xi) \tilde{f}_{j, 0}^{+}(t, \xi) d \xi=\int_{M} \psi(\xi) \exp \left(i \tau_{j}(\xi) t\right) \tilde{f}_{j, 0}^{+}(0, \xi) d \xi
$$

Hence for $f \in V$

$$
(U(t) f)_{+}(t, \xi)=\sum_{j=1}^{k} \exp \left(i \tau_{j}(\xi) t\right)\left(\tilde{f}_{+}(\xi) \cdot \overline{r_{j}(\xi)}\right) r_{j}(\xi)
$$

a.e. in $\xi$.

Moreover, given $f \in D_{-} \cap V$, (2.11) implies $\tilde{f}_{+}(\xi)=\hat{f}(\xi)$, and by construction $D_{-} \cap V$ is dense in $D_{-}$. Thus by (2.17) for $f \in D_{-} \cap V,\left(U_{0}(t)\right) f^{\wedge}(t, \xi)$ $=(U(t)) f_{+}^{\sim}(t, \xi)$, and $\mathscr{F}_{+}$extends to an isometry on $H_{-}$. Since the Fourier transform is a unitary map of $L^{2}$ onto $L^{2}$, it suffices to show $\left\{U_{0}(t) D_{-} \mid t \in \mathbf{R}\right\}$ is dense in $L^{2}$ to complete the proof. However,

$$
\left(R U_{0}(t) f\right)(s, \omega)=\sum_{j=1}^{k}\left(R f\left(s+\tau_{j}(\omega) t\right)\right) \cdot\left(\overline{r_{j}(\omega)}\right) r_{j}(\omega)
$$


and, by Lemma 2.2 of [7], $R^{-1}$ maps the even functions in $L^{2}\left(\mathbf{R}, L^{2}\left(S^{n-1}\right)\right)$ onto $L^{2}\left(\mathbf{R}^{n}\right)$. Since $\Pi_{j=1}^{k} \tau_{j}(\omega)=\operatorname{det} A_{0}(\omega) \neq 0$ on $S_{0}^{c}$ and $S_{0}$ is a closed set of spherical measure zero, the desired conclusion follows easily.

The next theorem is the main ingredient in the proof of the existence of the scattering matrix.

Theorem 2.2. Let $P_{ \pm}$be the projection on $D_{ \pm}^{\perp}$. Then, for $f$ in $H_{-}$or $H_{+}$, $\left\|P_{ \pm} U(t) f\right\|_{0} \rightarrow 0$ as $t \rightarrow \pm \infty$.

Proof. As before we only consider $f \in H_{-}$. Since $C_{0}^{\infty}(M)$ is dense in $L^{2}\left(\mathbf{R}^{n}\right)$, Theorem 2.1 implies $W \equiv \mathscr{F}_{+}^{*}\left(C_{0}^{\infty}(M)\right)$ is dense in $H_{-}$. We begin by showing $\left\|P_{-} P_{+} U(t) f\right\|_{0} \rightarrow 0$ as $t \rightarrow \pm \infty$.

For $f \in V \cap H_{-}$and $q \in C_{0}^{\infty}(M),(2.17)$ implies

$$
\begin{aligned}
&\left(U(t) \mathscr{F}_{+}^{*} q, f\right)=\left(q, \mathscr{F}_{+} U(-t) f\right)=\sum_{j=1}^{k} \int\left(q(\xi) \cdot \overline{r_{j}(\xi)} \exp \left(i \tau_{j}(\xi) t\right)\right) r_{j}(\xi) \cdot \overline{\mathscr{F}_{+} f(\xi)} d \xi \\
&= \sum_{j=1}^{k} \int\left(q(\xi) \cdot \overline{r_{j}(\xi)} \exp \left(i \tau_{j}(\xi) t\right)\right) \overline{\hat{f}(\xi)} \cdot r_{j}(\xi) d \xi \\
&+\sum_{j=1}^{k}(2 \pi)^{-n / 2} \int\left(q(\xi) \cdot \overline{\eta_{j}}(\xi) \exp \left(i \tau_{j}(\xi) t\right)\right) \\
& \cdot\left(\int_{\mathbf{R} \times S^{n-1}} \overline{R f} \cdot R v_{j}^{+}(s, \omega, \xi) d \sigma d \omega\right) d \xi \quad \text { by }(2.14) \\
&=(2 \pi)^{-n / 2} \int_{\mathbf{R}^{n}}\left(\sum_{j=1}^{k} \int_{\mathbf{R}^{n}}\left(q(\xi) \cdot \overline{r_{j}(\xi)}\right) \exp \left(i\left(\xi \cdot x+\tau_{j}(\xi) t\right)\right) d \xi\right) \cdot \overline{f(x)} d x \\
&+(2 \pi)^{-n / 2} \int_{\mathbf{R} \times S^{n-1}}\left(\int_{\mathbf{R}^{n}} \sum_{j=1}^{k} q(\xi) \cdot \overline{r_{j}(\xi)} \exp \left(i \tau_{j}(\xi) t\right) R v_{j}^{+}(s, \omega, \xi) d \xi\right)
\end{aligned}
$$

by Fubini's theorem, since $R v_{j}^{+} \in L_{\text {loc }}^{2}\left(\mathbf{R} \times S^{n-1} \times M\right)$. Hence, since $\{R f \mid f$ $\left.\in V \cap H_{-}\right\}$is dense in $R H_{-}$,

$$
\begin{aligned}
(2 \pi)^{n / 2} R U(t) \mathscr{F}_{+}^{*} q= & R\left(\sum_{j=1}^{k} \int_{\mathbf{R}^{n}}\left(q(\xi) \cdot \overline{r_{j}(\xi)}\right) \exp \left(i\left(\xi \cdot x+\tau_{j}(\xi) t\right)\right) r_{j}(\xi) d \xi\right) \\
& +\left(\sum_{j=1}^{k} \int_{\mathbf{R}^{n}}\left(q(\xi) \cdot \overline{r_{j}(\xi)} \exp \left(i \tau_{j}(\xi) t\right)\right) R v_{j}^{+}(s, \omega, \xi) d \xi\right)
\end{aligned}
$$

and, letting $b(x)=\int q(\xi) e^{i \xi \cdot x} d \xi$, we have

$$
\begin{aligned}
(2 \pi)^{n / 2} R U(t) \mathscr{F}_{+}^{*} q= & {\left[\left(\sum_{j=1}^{k} R b\left(s+\tau_{j}(\omega) t, \omega\right) \cdot \overline{r_{j}(\omega)} r_{j}(\omega)\right)\right] } \\
& +\left[\sum_{j=1}^{k} \int_{\mathbf{R}^{n}}\left(q(\xi) \cdot \overline{r_{j}(\xi)} \exp \left(i \tau_{j}(\xi) t\right)\right) \overline{R v_{j}^{+}(s, \omega, \xi)} d \xi\right]
\end{aligned}
$$


Since $R b(s, \omega)$ is an element of $L^{2}\left(\mathbf{R} \times S^{n-1}\right)$, the $L^{2}$-norm of the restriction of the first term on the right of $(2.18)$ to $\{(s, \omega)|| s \mid<R\}$ tends to zero as $t \rightarrow \pm \infty$. To show the same is true for the second term it suffices to show for $j=1, \ldots, k$

$$
0=\lim _{t \rightarrow \pm \infty}\left\|\int_{\mathbf{R}^{n}}\left(q(\xi) \cdot \overline{r_{j}(\xi)} \exp \left(i \tau_{j}(\xi) t\right)\right) R v_{j}^{+}(s, \omega, \xi) d \xi\right\|_{\left.L^{2}(|| s \mid<R\} \times S^{n-1}\right)} .
$$

This will be a consequence of our estimates on $R v_{j}^{+}$, and a number of applications of the Riemann-Lebesgue lemma and the Lebesgue dominated convergence theorem. Introducing polar coordinates in $\xi$-space, the function of (2.19) becomes

$$
H(s, \omega, t)=\int_{S^{n-1}} d \omega^{\prime} \int_{\mathbf{R}}\left(q\left(\sigma \omega^{\prime}\right) \cdot \overline{r_{j}\left(\omega^{\prime}\right)} \exp \left(i \sigma \tau_{j}\left(\omega^{\prime}\right) t\right)\right) R v_{j}^{+}\left(s, \omega, \sigma \omega^{\prime}\right) \sigma^{n-1} d \sigma .
$$

Since $q(\xi) \in C_{0}^{\infty}$ and $\left|\tau_{j}(\xi /|\xi|)\right| \geq \delta>0$ on support $q$, the Riemann-Lebesgue lemma implies for any sequence $t_{i} \rightarrow \pm \infty$ the inner integral of (2.20) tends to zero a.e. in $\omega^{\prime}, s, \omega$. Hence by Lebesgue dominated convergence $H\left(s, \omega, t_{i}\right) \rightarrow 0$ a.e. as $i \rightarrow \infty$. However, since $\left|R v_{j}^{+}(s, \omega, \xi)\right| \leq C$ for $(s, \omega) \in \mathbf{R} \times S^{n-1}$ and $\xi$ in support $q$, the restriction of $H\left(s, \omega, t_{i}\right)$ to $|s|<R$ is uniformly bounded by a constant multiple of the characteristic function of $\{s|| s \mid<R\}$ and another application of L.D.C. gives (2.19). Thus, for $f \in W,\left\|P_{-} P_{+} U(t) f\right\|_{0} \rightarrow 0$ as $t \rightarrow \pm \infty$.

From this point the proof may be identified with a subset of the proof of $[8$, Lemma 3.8]. For $f \in W$ we consider $R(U(t) f)$. Since $U(t) f$ is a distribution solution to $\left(\partial / \partial t-L_{0}\right) U(t) f=\left(L-L_{0}\right) U(t) f$, and $\left(L-L_{0}\right) U(t) f$ is supported in $|x|<R$, we conclude $R(U(t) f)$, is a distribution solution to $(\partial / \partial t$ $\left.-A_{0}(\omega) \partial / \partial s\right) R(U(t) f)=0$ in $|s|>R$. Hence

$$
\sum_{k=1}^{k(\omega)}\left((R U(t) f)(s, \omega) \cdot \overline{r_{l}(\omega)}\right) r_{l}(\omega)=\sum_{l=1}^{k(\omega)}\left(R f\left(s+\tau_{l}(\omega) t, \omega\right) \cdot \overline{r_{l}(\omega)}\right) r_{l}(\omega)
$$

for $t<0, s<-R$, and for $t>0, s>R$. Likewise for $t>0, s<-R$, and $t<0, s>R$

$$
\sum_{l=k(\omega)+1}^{k}\left((R U(t) f)(s, \omega) \cdot \overline{r_{l}(\omega)}\right) r_{l}(\omega)=\sum_{l=k(\omega)+1}^{k}\left((R f)\left(s+\tau_{l}(\omega) t, \omega\right) \cdot \overline{r_{l}(\omega)}\right) r_{l}(\omega) .
$$

Since $f$ and hence $R f$ are square-integrable, $\prod_{j=1}^{k} \tau_{j}(\omega)=\operatorname{det} A_{0}(\omega) \neq 0$ on $S_{0}^{c}$, and $S_{0}$ is a closed set of spherical measure zero, it follows $\left\|P_{D_{-}} U(t) f\right\|_{0} \rightarrow 0$ as $t \rightarrow \infty$ and $\left\|P_{D_{+}} U(t) f\right\|_{0} \rightarrow 0$ as $t \rightarrow-\infty$. Here $P_{D_{ \pm}}$is the projection on $D_{ \pm}$. Combining this with $\left\|P_{-} P_{+} U(t) f\right\|_{0} \rightarrow 0$ as $t \rightarrow \pm \infty$, completes the proof of Theorem 2.2.

3. Unitarity of the scattering matrix. We begin by constructing a pseudodifferential operator-valued function of $t, Q(t)$. The use of $Q(t)$ in this section 
could probably be avoided by another application of Hörmander's Proposition 3.5.1 but $Q(t)$ seems essential for the proof of Theorem 4.1. Our construction was suggested by that of $[3, \S 2.4]$.

The symbol of $Q(t)$ will be given by $a(x, \xi, t) P(x, \xi)+Q_{-1}(x, \xi, t) / i \equiv Q_{0}$ $+Q_{-1} / i$, where $Q_{j}$ is homogeneous degree $j$ and $P(x, \xi)$ is projection operator of $\S 1$.

We want $[\partial / \partial t-L, Q(t)]$ to be an operator of order -1 . Thus we require

$$
\begin{aligned}
A(x, \xi) & Q_{-1}(x, \xi, t)-Q_{-1}(x, \xi, t) A(x, \xi) \\
= & \frac{\partial a}{\partial t} P+\sum_{i=1}^{n}\left(\frac{\partial Q_{0}}{\partial \xi_{i}} \frac{\partial A}{\partial x_{i}}-\frac{\partial A}{\partial \xi_{i}} \frac{\partial Q_{0}}{\partial x_{i}}\right)+Q_{0} B-B Q_{0} \equiv H .
\end{aligned}
$$

Let $P^{\prime}=1-P$. As a first step in the solution of (3.1) for $Q_{-1}$, we show

$$
{ }^{\prime} Q_{-1}^{\prime} \equiv \sum_{i=1}^{n} \frac{\partial P^{\prime}}{\partial \xi_{i}} Q_{0} \frac{\partial P^{\prime}}{\partial x_{i}}
$$

is a smooth solution to

$$
A^{\prime} Q_{-1}^{\prime}-{ }^{\prime} Q_{-1}^{\prime} A=P^{\prime} H P^{\prime}
$$

We have

$$
\begin{aligned}
P^{\prime} H P^{\prime} & =\sum_{i=1}^{n} P^{\prime} \frac{\partial Q_{0}}{\partial \xi_{i}} \frac{\partial A}{\partial x_{i}} P^{\prime}-P^{\prime} \frac{\partial A}{\partial \xi_{i}} \frac{\partial Q_{0}}{\partial x_{i}} P^{\prime} \\
& =\sum_{i=1}^{n}\left[-\frac{\partial P^{\prime}}{\partial \xi_{i}} Q_{0} \frac{\partial A}{\partial x_{i}} P^{\prime}+P^{\prime} \frac{\partial A}{\partial \xi_{i}} Q_{0} \frac{\partial P^{\prime}}{\partial x_{i}}\right],
\end{aligned}
$$

since $P^{\prime} P=0$.

$$
P^{\prime} H P^{\prime}=\sum_{i=1}^{n}-\frac{\partial P^{\prime}}{\partial \xi_{i}}\left(-\frac{\partial Q_{0}}{\partial x_{i}} A+\frac{\partial \tau Q_{0}}{\partial x_{i}}\right) P^{\prime}+P^{\prime}\left(-A \frac{\partial Q_{0}}{\partial \xi_{i}}+\frac{\partial \tau Q_{0}}{\partial \xi_{i}}\right) \frac{\partial P^{\prime}}{\partial x_{i}},
$$

since $Q_{0} A-\tau Q_{0}=A Q_{0}-\tau Q_{0}=0$.

$$
\begin{aligned}
P^{\prime} H P^{\prime}= & \sum_{i=1}^{n} \frac{\partial P^{\prime}}{\partial \xi_{i}} \frac{\partial Q_{0}}{\partial x_{i}} P^{\prime} A-A P^{\prime} \frac{\partial Q_{0}}{\partial \xi_{i}} \frac{\partial P^{\prime}}{\partial x_{i}} \\
& +\sum_{i=1}^{n} \tau\left(-\frac{\partial P^{\prime}}{\partial \xi_{i}} \frac{\partial Q_{0}}{\partial x_{i}} P^{\prime}+P^{\prime} \frac{\partial Q_{0}}{\partial \xi_{i}} \frac{\partial P^{\prime}}{\partial x_{i}}\right) \\
= & \sum_{i=1}^{n}-\frac{\partial P^{\prime}}{\partial \xi_{i}} Q_{0} \frac{\partial P^{\prime}}{\partial x_{i}} A+A \frac{\partial P^{\prime}}{\partial \xi_{i}} Q_{0} \frac{\partial P^{\prime}}{\partial x_{i}}
\end{aligned}
$$

Hence ' $Q_{-1}^{\prime}=\sum_{i=1}^{n} \partial P^{\prime} / \partial \xi_{i} Q_{0} \partial P^{\prime} / \partial x_{i}$ is a solution to (3.2) as claimed. If $P H P=0$, there is a solution ' $Q_{-1}$ to $(A-\tau I)^{\prime} Q_{-1}=H P$ satisfying ' $Q_{-1} P^{\prime}=0$. Hence $A^{\prime} Q_{-1}{ }^{\prime} Q_{-1} A=H P$. Likewise, if $P H^{*} P=0$, there is a solution $Q_{-1}^{\prime}$ to $Q_{-1}^{\prime}(\tau I-A)=P H$ satisfying $P^{\prime} Q^{\prime}=0$. Hence $A Q_{-1}^{\prime}-Q_{-1}^{\prime} A=P H$. A com- 
putation similar to that following (3.2) shows both $P H P=0$ and $P H^{*} P=0$ reduce to

$$
0=\frac{\partial a}{\partial t}+\sum_{i=1}^{n} \frac{\partial a}{\partial \xi_{i}} \frac{\partial \tau}{\partial x_{i}}-\frac{\partial a}{\partial x_{i}} \frac{\partial \tau}{\partial \xi_{i}}
$$

Hence, if (3.3) holds we may solve (3.1) with $Q_{-1}={ }^{\prime} Q_{-1}^{\prime}+Q_{-1}^{\prime}+{ }^{\prime} Q_{-1}$ and $Q_{-1}$ will be a symbol of order -1 depending smoothly on $t$. Given a bicharacteristic $(x(t), \xi(t))$ satisfying $\dot{x}=-\partial \tau / \partial \xi$ and $\dot{\xi}=\partial \tau / \partial x,(3.3)$ merely states

$$
0=d a(x(t), \xi(t), t) / d t .
$$

Thus we choose $a(x, \xi, t)$ constant on the curves $(x(t), \xi(t), t)$. If we choose $a(x, \xi, 0)=1$ on $S_{R}$ with $a(x, \xi, 0)=0$ outside a sufficiently small neighborhood of $S_{R}$ and determine $a$ for $t>0$ by (3.4), it follows by hypothesis (ii) that there is a $T_{0}$ such that $a\left(x, \xi, T_{0}\right)=0$ for $|x|<R+1$. We extend $a(x, \xi, t)$ to be homogeneous degree 0 in $\xi$ for $|\xi|>1$ and smooth in $\xi$ on $\mathbf{R}^{n}$. The symbol $Q_{-1}(t, x, \xi)$ may be chosen to have support contained in the support of $a$.

We form $Q(t)$ with symbol $a P+Q_{-1} / i$ constructed in the preceding paragraph and given $R^{\prime}$, have $\|[\partial / \partial t-L, Q(t)] u\|_{1}^{R^{\prime}} \leq C_{R^{\prime}}\|u\|_{0}$ for all $u \in C_{0}^{\infty}\left(|x|<R^{\prime}\right)$, where $C_{R^{\prime}}$ is independent of $t$ for $t \in\left[0, T_{0}\right]$. Moreover, multiplying $Q(t)$ on the right by $\psi(x, t), \psi(x, t)=0$ outside small neighborhood of support $a(x, \cdot, t), \psi(x, t)$ $=1$ on support $a(x, \cdot, t)$, we may assume $Q\left(T_{0}\right) u=0$ for all $u \in C_{0}^{\infty}(|x|<R)$, and $Q(t) u$ has compact support for all $t$.

We are now ready to complete the proof of the unitarity of the scattering matrix and energy decay. The following lemma is patterned on Theorem 1 of [9].

Lemma 3.1. $H_{+}+H_{-}$is dense in $L^{2}\left(\mathbf{R}^{n}\right) \ominus H_{0}$.

Proof. Suppose we have $q \in L^{2}\left(\mathbf{R}^{n}\right)$ such that $q \perp\left(H_{0}+H_{+}+H_{-}\right)$. Then, by the definition of $H_{+}$and $H_{-}, U(t) q \perp D_{+} \forall t$ and $U(t) q \perp D_{-}$all $t$. Hence $R(U(t) q)(s, \omega)=0,|s|>R, \forall t$. By domain of dependence $U_{0}\left(t-t_{0}\right) U\left(t_{0}\right) q$ $=U(t) q$ for $|x|>R+2 c \delta$ and $\left|t-t_{0}\right|<\delta$, where $c$ is the maximum sound speed of $L, c=\sup _{x \in \mathbf{R}^{n}}\{v \cdot A(x, \xi) v|| \xi|=1| v \mid,=1\}$. Let

$$
h(r) \in\left(C_{0}^{\infty}(R+2 c \delta, \infty)\right)^{k}
$$

and consider

$$
m(s, \omega)=\int_{\omega \cdot x=s} Y_{m}\left(\frac{x}{|x|}\right) h(|x|) d A
$$

where $Y_{m}$ is a spherical harmonic of degree $m$. Rotating coordinates so that $\omega \cdot x=y_{1}$

$$
m(s, \omega)=\sum_{|\alpha|=m} a_{\alpha}(\omega) \int_{y_{1}=s} \frac{y^{\alpha}}{|y|^{m}} h(|y|) d y_{2} d y_{n}
$$


Introducing polar coordinates for $y_{2}, \ldots, y_{n}$ and setting $\alpha=\left(\alpha_{1}, \alpha^{\prime}\right)$

$$
m(s, \omega)=\sum_{|\alpha|=m} a_{\alpha}(\omega) s^{\alpha_{1}} \int_{S^{n-2}} d \omega^{\prime} f_{\alpha}\left(\omega^{\prime}\right) \int_{0}^{\infty}\left(r^{2}+s^{2}\right)^{-m / 2} h\left(\left(r^{2}+s^{2}\right)^{1 / 2}\right) r^{n+\left|\alpha^{\prime}\right|-2} d r
$$

Now if $|s|<R+c \delta$ the $r$-integration does not include $r=0$ and we can introduce $u=\left(r^{2}+s^{2}\right)^{1 / 2}$ and get

$$
m(s, \omega)=\sum_{|\alpha|=m} b_{\alpha}(\omega) s^{\alpha_{1}} \int_{\mathbf{R}+2 c \delta}^{\infty} u^{(1-m)} h(u)\left(u^{2}-s^{2}\right)^{\left(n+\left|\alpha^{\prime}\right|-3\right) / 2} d u .
$$

Now let $n\left(s, \omega, t_{0}\right)$ be the Radon transform of $U\left(t_{0}\right) q$. Then the Radon transform of $U_{0}\left(t-t_{0}\right) U\left(t_{0}\right) q$ is

$$
\sum_{j=1}^{n} n_{j}\left(s+\tau_{j}(\omega)\left(t-t_{0}\right), \omega, t_{0}\right) r_{j}(\omega)
$$

(here $\left.n_{j}=n\left(s, \omega, t_{0}\right) \cdot \overline{r_{j}(\omega)}\right)$. Letting

$$
l_{j}\left(s, \omega, t_{0}\right)=\left(\frac{\partial}{\partial s}\right)^{(n-1 / 2)} n_{j}(s, \omega, t) \text { and } k_{j}(s, \omega)=\left(\frac{\partial}{\partial s}\right)^{(n-1 / 2)} m_{j}(s, \omega),
$$

we have

$$
\begin{aligned}
C(t) & =\int h(x) Y_{m}\left(\frac{x}{|x|}\right) \overline{(U(t) q)(x)}=\int R\left(h Y_{m}\right) \overline{R(U(t) q)} \\
& =\sum_{j=1}^{k} \int_{S^{n-1}} d \omega \int_{\left|s-\tau_{j}(\omega)\left(t-t_{0}\right)\right| \leq R} k_{j}(s, \omega) \bar{l}_{j}\left(s+\tau_{j}(\omega)\left(t-t_{0}\right), \omega, t_{0}\right) d s \\
& =\sum_{j=1}^{k} \int_{S^{n-1}} d \omega \int_{|v| \leq R} k_{j}\left(v-\tau_{j}(\omega)\left(t-t_{0}\right), \omega\right) \bar{l}_{j}\left(v, \omega, t_{0}\right) d v
\end{aligned}
$$

and by (3.5) for $\left|\operatorname{Re}\left(t-t_{0}\right)\right|<\delta$ we have $C(t)$ analytic. Since $t_{0}$ was arbitrary, $C(t)$ is entire. Moreover, since the norm of $l\left(s, \omega, t_{0}\right)$ in $L^{2}\left(\mathbf{R} \times S^{n-1}\right)$ is constant and

$$
\sup _{|v| \leq R ;|\omega|=1}\left|k_{j}\left(v-\tau_{j}(\omega)\left(t-t_{0}\right)\right)\right| \leq C\left|t-t_{0}\right|^{(n-5) / 2+m} \leq C(|\operatorname{Im} t|+\delta)^{(n-5) / 2+m},
$$

we conclude $C(t)$ is a polynomial. Since $C(t)$ is bounded on the real axis, $C(t)$ is constant. Thus $U(t) q(x)$ is constant for $|x|>R$.

Let $h=U\left(t_{1}\right) q-U\left(t_{2}\right) q$. Then $U(t) h=0$ for $|x|>R$. If $h$ is not zero, neither is $\varphi=n \int_{0}^{1 / n} U(t) h d t$ for $n$ sufficiently large. Moreover, $\varphi \in \mathcal{D}(L)$ and $U(t) \varphi$ $=0$ for $|x|>R$.

Now let $Q(t)$ be the operator-valued function constructed at the beginning of this section. We denote the operator $-[\partial / \partial t-L, Q(t)]$ by $K(t)$. Then we have 
$\partial Q\left(t-t_{0}\right) U(t) \varphi / \partial t-L\left(Q\left(t-t_{0}\right) U(t) \varphi\right)=K\left(t-t_{0}\right) U(t) \varphi$ and by Duhamel's formula

$$
Q\left(t-t_{0}\right) U(t) \varphi=U\left(t-t_{0}\right) Q(0) U\left(t_{0}\right) \varphi+\int_{t_{0}}^{t} U(t-s) K\left(s-t_{0}\right) U(s) \varphi d s
$$

For all real $s, U(t)$ satisfies the estimate

$$
\|U(t) f\|_{s} \leq C_{s} e^{\omega_{s}|t|}\|f\|_{s}
$$

To see this, assume first $s \leq 0$, and let $\Lambda_{s}$ denote the operator on $L^{2}\left(\mathbf{R}^{n}\right)$ which multiplies the Fourier transform by $\left(1+|\xi|^{2}\right)^{s / 2}$. Then for $f \in \mathscr{D}(L)$

$$
\frac{\partial \Lambda_{s} U(t) f}{\partial t}=L \Lambda_{s} U(t) f+\left[\Lambda_{s}, L\right] \Lambda_{s}^{-1} \Lambda_{s} U(t) f
$$

Since $\left[\Lambda_{s}, L\right] \Lambda_{s}^{-1}=\left[\Lambda_{s}, L-L_{0}\right] \Lambda_{s}^{-1}$ is an operator of order zero, and, for $|x|$ $>R,\left(\left[\Lambda_{s}, L\right] \Lambda_{s}^{-1} f\right)(x)=\left(\Lambda_{s}\left(L-L_{0}\right) \Lambda_{s}^{-1} f\right)(x),\left[\Lambda_{s}, L\right] \Lambda_{s}^{-1}$ is a bounded operator on $L^{2}\left(\mathbf{R}^{n}\right)$. Thus $L+\left[\Lambda_{s}, L\right] \Lambda_{s}^{-1}$ is a bounded perturbation of the group generator $L$, and therefore generates an exponentially bounded group $\tilde{U}(t)$. Since $\Lambda_{s} U(t) f$ is continuously strongly differentiable, (3.8) implies $\Lambda_{s} U(t) f=\tilde{U}(t) \Lambda_{s} f$ and we have

$$
\left\|\Lambda_{s} U(t) f\right\|_{0} \leq C_{s} e^{\omega_{s}|t|}\left\|\Lambda_{s} f\right\|_{0} .
$$

Since $C_{0}^{\infty}\left(\mathbf{R}^{n}\right) \subset \mathscr{D}(L)$ is dense in $H_{s}$, (3.7) follows immediately from (3.9).

For $s>0$ we argue by duality:

$$
\begin{aligned}
\|U(t) f\|_{s} & =\sup _{g \in C_{0}^{\infty} ;\|g\|-s \leq 1}\left|\int \bar{g} U(t) f\right|=\sup \left|\int \overline{U(-t) g} f\right| \\
& \leq C_{-s} e^{\omega-s|t|} \sup _{g \in C_{0}^{\infty} ;\|g\|_{s} \leq 1}\left|\int \bar{g} f\right|=C_{-s} e^{\omega_{s}|t|}\|f\|_{s} .
\end{aligned}
$$

Thus we have (3.7) for all $s$.

Since $\left\|K\left(s-t_{0}\right) U(s) \varphi\right\|_{h} \leq C\|\varphi\|_{0}$, for $t_{0} \leq s \leq T_{0}+t_{0}$, we conclude from (3.6) and (3.7) with $s=1$

$$
\left\|Q(0) U\left(t_{0}\right) \varphi\right\|_{1} \leq K_{1}\left\|U\left(T_{0}\right) Q(0) U\left(t_{0}\right) \varphi\right\|_{1} \leq K_{2}\left(\left\|Q\left(T_{0}\right) U\left(t_{0}+T_{0}\right) \varphi\right\|_{1}+\|\varphi\|_{0}\right) .
$$

$U\left(t_{0}+T_{0}\right) \varphi=0$ for $|x|>R$ and by construction $Q\left(T_{0}\right) U\left(t_{0}+T_{0}\right) \varphi=0$. Hence $\|Q(0) U(t) \varphi\|_{1} \leq K_{2}\|\varphi\|_{0}=C_{0}$. Since $\varphi \in \mathscr{D}(L), \quad\|L U(t) \varphi\|_{0}=\|U(t) L \varphi\|_{0}$ $\leq\|L \varphi\|_{0}=C_{1}$. We may choose $\alpha \in C_{0}^{\infty}, \alpha=1$ for $|x|<R$, such that (compare (1.14)) $\|\alpha \psi\|_{1} \leq C\left(\|L \psi\|_{0}+\|Q(0) \psi\|_{1}\right)$. Thus we have

$$
\|U(t) \varphi\|_{1}=\|\alpha U(t) \varphi\|_{1} \leq C\left(C_{1}+C_{0}\right) \quad \forall t,
$$

and $\{U(t) \varphi \mid t \in \mathbf{R}\}$ is a precompact set in $L^{2}\left(\mathbf{R}^{n}\right)$. Since $\varphi \perp H_{0}$, Lemma V.2.3 
of [6] implies there are $T_{i} \rightarrow \infty$ such that $U\left(T_{i}\right) \varphi$ converges weakly to zero. By compactness $\left\|U\left(T_{i_{k}}\right) \varphi\right\|_{0} \rightarrow 0$ for a subsequence $T_{i_{k}}$ and $\varphi=0$. Thus $U(t) q$ $=q \forall t$ and, since $q \perp H_{0}, q=0$.

We define wave operators $W_{+}$and $W_{-}$by

$$
W_{ \pm} f=\lim _{t \rightarrow \pm \infty} U(-t) U_{0}(t) f .
$$

These limits exist for $f \in\left\{U_{0}(t) D_{ \pm} \mid t \in \mathbf{R}\right\}$. Since the argument following (2.17) shows $\left\{\overline{U_{0}(t) f \mid f \in D_{+}}\right\}=\left\{\overline{U_{0}(t) f \mid f \in D_{-}}\right\}=L^{2}\left(\mathbf{R}^{n}\right), W_{+}$and $W_{-}$are isometries from $L^{2}\left(\mathbf{R}^{n}\right)$ into $L^{2}\left(\mathbf{R}^{n}\right)$.

Theorem 3.2. Range $W_{+}=$Range $W_{-}=L^{2}\left(\mathbf{R}^{n}\right) \ominus H_{0}$.

Proof. The proofs for $W_{+}$and $W_{-}$are the same; we give the proof for $W_{+}$.

Let $h=q_{1}+q_{2}, q_{1} \in H_{-}, q_{2} \in H_{+}$. Then, by Theorem 2.2, $\left\|P_{+} U(t) h\right\|_{0}<\varepsilon$ for $t \geq T$. Letting $\left(I-P_{+}\right) U(T) h=f$ we have for $t>0$

$$
\begin{array}{r}
\left\|U_{0}(t) f-U(t+T) h\right\|_{0}<\varepsilon, \\
\left\|U(-t-T) U_{0}(t+T)\left[U_{0}(-T) f\right]-h\right\|_{0}<\varepsilon, \\
\left\|W_{+} U_{0}(-T) f-h\right\|_{0}<\varepsilon .
\end{array}
$$

Since $\varepsilon$ is arbitrary and the range of $W_{+}$is closed, we have $h \in$ Range $W_{+}$. By Lemma 3.1, Range $W_{+} \subset L^{2}\left(\mathbf{R}^{n}\right) \ominus H_{0} . f \in$ Range $W_{+} \cap H_{0} \Rightarrow\left\|e^{i \lambda t} f-U_{0}(t) h\right\|_{0} \rightarrow$ 0 for some $h \in L^{2}\left(\mathbf{R}^{n}\right)$. Since $\left\|U_{0}(t) h\right\|_{0}^{r} \rightarrow 0$ as $t \rightarrow \infty$ for all $r$ (again see argument following (2.17)), we conclude $f=0$.

Corollary 3.3. $\mathrm{H}^{+}=\mathrm{H}^{-}=L^{2}\left(\mathbf{R}^{n}\right) \ominus H_{0}$. Hence $\mathscr{F}_{+}$and $\mathscr{F}_{-}$extend to unitary maps of $L^{2}\left(\mathbf{R}^{n}\right) \ominus H_{0}$ to $L^{2}\left(\mathbf{R}^{n}\right)$.

Corollary 3.4. The scattering matrix $S=W_{-}^{*} W_{+}$is unitary.

Corollary 3.5. For $f \in L^{2}\left(\mathbf{R}^{n}\right)-H_{0}$ and $r<\infty$,

$$
\|U(t) f\|_{0}^{r} \rightarrow 0, \quad t \rightarrow \pm \infty .
$$

Proof. By Theorem $3.2 \exists h_{ \pm} \in L^{2}(\mathbf{R})$ such that $\left\|U(t) f-U_{0}(t) h_{ \pm}\right\|_{0} \rightarrow 0$ as $t \rightarrow \pm \infty$ and $\left\|U_{0}(t) h_{ \pm}\right\|_{0}^{r} \rightarrow 0$ as $t \rightarrow \pm \infty$.

4. Concentration near bicharacteristics and an example of nondecay. In this section we assume the existence of a smooth real-valued function $\tau(x, \xi)$ and a smooth projection-valued function $P(x, \xi)$, defined on a conic open set $V$ in $(x, \xi)$-space, such that $A(x, \xi) P(x, \xi)=\tau(x, \xi) P(x, \xi)$. Given a fixed $\lambda_{0} \in \mathbf{R}$, we define the level set $S_{\lambda_{0}}$ to be $\left\{(x, \xi) \in V \mid \tau(x, \xi)=\lambda_{0}\right\}$ if $\lambda_{0} \neq 0$ and define $S_{\lambda_{0}}=\{(x, \xi) \in V|\tau(x, \xi)=0,| \xi \mid=1\}$ if $\lambda_{0}=0$. We assume that $S_{\lambda_{0}}$ is closed in $(x, \xi)$-space and the null space of $A(x, \xi)-\lambda_{0} I$ is one dimensional for $(x, \xi) \in S_{\lambda_{0}}$. Let $x(t), 0 \leq t \leq T$, be the curve determined by 


$$
\dot{x}=-\partial \tau / \partial \xi, \quad \dot{\xi}=\partial \tau / \partial x,
$$

where $(x(0), \xi(0))=\left(x_{0}, \xi_{0}\right) \in S_{\lambda_{0}}$. Then we have

Theorem 4.1. Given neighborhoods of $x_{0}$ and $x_{T}=x(T), N_{0}$ and $N_{T}$, there is a $\varphi_{\varepsilon} \in C_{0}^{\infty}\left(N_{0}\right)$ such that $\left\|\varphi_{\varepsilon}\right\|_{0}=1$ and $\left\|U(t) \varphi_{\varepsilon}\right\|_{0}^{N_{T}} \geq 1-\varepsilon$ for all $\varepsilon>0$.

Proof. We build $Q(t), 0 \leq t \leq T$, as in $\S 3$, but here $a(x, \xi, 0)$ is chosen with support in a sufficiently small conic neighborhood of $\left(x_{0}, \xi_{0}\right)$ that the support of $a(x, \xi, 0)$ in $x$ is contained in $N_{0}$ and the support of $a(x, \xi, T)$ in $x$ is contained in $N_{T} . a(x, \xi, 0)$ is chosen equal 1 on a conic neighborhood of $\left(x_{0}, \xi_{0}\right)$.

Let $\varphi(x)=\psi(x) e^{i k \xi_{0} \cdot x}$ where $\psi \in C_{0}^{\infty}\left(N_{0}\right)$. Since $\varphi \in \mathscr{D}(L)$ we have

$$
\frac{\partial Q(t) U(t) \varphi}{\partial t}-L Q(t) U(t) \varphi=K(t) U(t) \varphi
$$

and $K(t)$ is a uniformly (for $0 \leq t \leq T$ ) bounded operator from $H_{-1}\left(\mathbf{R}^{n}\right)$ to $L^{2}\left(\mathbf{R}^{n}\right)$. By Duhamel's formula

$$
\begin{aligned}
Q(T) U(T) \varphi-U(T) Q(0) \varphi & =\int_{0}^{T} U(T-s) K(s) U(s) \varphi d s \\
\|Q(T) U(T) \varphi-U(T) Q(0) \varphi\|_{0} & \leq T \sup _{t \in[0, T]}\|K(t) U(t) \varphi\|_{0} \leq C_{T} \sup _{t \in[0, T]}\|U(t) \varphi\|_{-1} .
\end{aligned}
$$

Hence, using (3.7) with $s=-1$, we conclude

$$
\|Q(T) U(T) \varphi-U(T) Q(0) \varphi\|_{0} \leq K_{T}\|\varphi\|_{-1} .
$$

As $k \rightarrow \infty,\|\varphi\|_{-1} \rightarrow 0$. Since $Q(t)$ was constructed so that $Q(T) f$ vanishes outside a small neighborhood of the support of $a(x, \xi, T)$ in $x$, we may assume $Q(T) U(T) \varphi=0$ off $N_{T}$. Hence as $k \rightarrow \infty$

$$
\|U(T) Q(0) \varphi\|_{0}^{R^{n}-N_{T}} \rightarrow 0 .
$$

Since $U(t)$ is unitary on $L^{2}$, to prove Theorem 4.1 it suffices to show we can choose $\psi$ so that $\|Q(0) \varphi\|_{0} \nrightarrow 0$ as $k \rightarrow \infty$. The basic estimate from the symbol calculus of pseudo-differential operators gives

$$
e^{-i k x \cdot \xi_{0}} Q(0) \varphi \rightarrow a\left(x, \xi_{0}, 0\right) P\left(x, \xi_{0}\right) \psi(x)
$$

uniformly in $x$ over compact subsets as $k \rightarrow \infty$. Hence, we choose $\psi$ so that $P\left(x_{0}, \xi_{0}\right) \psi\left(x_{0}\right) \neq 0$ and then $\|Q(0) \varphi\|_{0} \nrightarrow 0$ as $k \rightarrow \infty$.

Theorem 4.1 has the following corollary.

Corollary 4.2. Let $\chi_{B}$ denote the characteristic function of $|x|<B$. If $|x(t)|<B$ for all $t>0$, the norm of the operator $\chi_{B} U(t) \chi_{B}$ is one for all $t \geq 0$.

Corollary 4.2 says no uniform decay estimate is possible if a simple bicharacteristic is trapped for $t>0$. However, if the bicharacteristic is not a null 
bicharacteristic and hypothesis (ii) holds, Corollary 3.4 still implies $\left\|\chi_{B} U(t) f\right\|_{0}$ $\rightarrow 0$ as $t \rightarrow \infty$ for all $f \in L^{2}\left(\mathbf{R}^{n}\right) \ominus H_{0}$. Our concluding example shows this can fail if there are trapped null bicharacteristics.

Example. We construct a vector field on $\mathbf{R}^{3}$ as follows: Let $\psi(r, s) \in C_{0}^{\infty}$ with $\psi(r, s)=0$ for $(r, s)$ outside $\{(r, s)|| s \mid \leq 1,1 \leq r \leq 2\}$. Assume $0 \leq \psi(r, s) \leq 1$, $\psi(r, 0)=1$ for $4 / 3 \leq r \leq 5 / 3$, and $\psi(s, r)<1$ for $s \neq 0$. Then we define for $r=\left(x_{1}^{2}+x_{2}^{2}\right)^{1 / 2}, \quad \mathbf{v}\left(x_{1}, x_{2}, x_{3}\right)=\psi\left(r, x_{3}\right)\left(-x_{2} / r, x_{1} / r, 0\right)+\left(1-\psi\left(r, x_{3}\right)\right)(0,0,1)$. Note that $\mathbf{v}(\mathbf{x})=(0,0,1)$ for $|\mathbf{x}|>\sqrt{ } 5$, and $|\mathbf{v}(\mathbf{x})|>0$ for all $\mathbf{x}$. Let $\mathbf{x}(t, y)$ be the solution to $\dot{\mathbf{x}}=\mathbf{v}(\mathbf{x}), \mathbf{x}(0, \mathbf{y})=\mathbf{y} \cdot r(t)=\left(x_{1}(t)^{2}+x_{2}(t)^{2}\right)^{1 / 2}=\left(y_{1}^{2}+y_{2}^{2}\right)^{1 / 2}$ for all $t$. Hence $x_{3}(t, \mathbf{y})$ is a nondecreasing function of $t$ with $x_{3}(t, \mathbf{y}) \rightarrow 0$ as $t \rightarrow \infty$ for $y_{3}<0,4 / 3 \leq\left(y_{1}^{2}+y_{2}^{2}\right) \leq 5 / 3$, and $x_{3}(t, \mathbf{y}) \rightarrow 0$ as $t \rightarrow-\infty$ for $y_{3}>0,4 / 3$ $\leq\left(y_{1}^{2}+y_{2}^{2}\right)^{1 / 2} \leq 5 / 3$.

Now let $U(t)$ be the unitary group of $L^{2}-\left(\mathbf{R}^{3}\right)$ given by

$$
(U(t) f)(\mathbf{y})=f(\mathbf{x}(t, \mathbf{y}))((\partial \mathbf{x} / \partial \mathbf{y})(t, \mathbf{y}))^{1 / 2} .
$$

By the preceding remarks, given $f(\mathbf{x})$ supported in $|x|<\sqrt{5}, x_{3}<0,4 / 3$ $<\left(x_{1}^{2}+x_{2}^{2}\right)^{1 / 2}<5 / 3,\|U(t) f\|_{0}^{\sqrt{ } 5}=\|f\|_{0}$ for $t>0$.

One computes $U(t)$ is generated by $L=\mathbf{v} \cdot \boldsymbol{\nabla}+\frac{1}{2} \boldsymbol{\nabla} \cdot \mathbf{v}$. Hence $A(x, \xi)$ $=\tau(x, \xi)=\mathbf{v}(\mathbf{x}) \cdot \xi$. The bicharacteristic through $(\mathbf{y}, \boldsymbol{\eta})$ has the form $(\mathbf{x}(t, \mathbf{y})$, $\boldsymbol{\xi}(t, \mathbf{y}, \boldsymbol{\eta}))$ where $\mathbf{x}(t, \mathbf{y})$ is function defined in the preceding paragraph. If $\mathbf{v}(\mathbf{y}) \cdot \boldsymbol{\eta}=0$, this is a null bicharacteristic. Thus every integral curve of $\mathbf{v}(\mathbf{x})$ is a null bicharacteristic for the proper choice of $\boldsymbol{\eta}$.

\section{REFERENCES}

1. D. M. Èìdus, The principle of limiting absorption, Mat. Sb. 57 (99) (1962), 13-44; English transl., Amer. Math. Soc. Transl. (2) 47 (1965), 157-191. MR 26 \#2722.

2. C. I. Goldstein, Eigenfunction expansions associated with the Laplacian for certain domains with infinite boundaries. I, Trans. Amer. Math. Soc. 135 (1969), 1-31. MR 38 \#2459.

3. L. Hörmander, Linear differential operators, Proc. Internat. Congress Math. (Nice, 1970), vol. 1, Gauthier-Villars, Paris, 1971, pp. 121-133.

4.—_ Fourier integral operators. I, Acta Math. 127 (1971), 79-183.

5.—, On the existence and regularity of solutions of linear pseudo-differential equations, Enseignement Math. 17 (1971), 99-163.

6. P. D. Lax and R. S. Phillips, Scattering theory, Pure and Appl. Math., vol. 26, Academic Press, New York, 1967. MR 36 \#530.

7.—, Scattering theory, Rocky Mountain J. Math. 1 (1971), 173-223.

8. - Scattering theory for the acoustic equation in an even number of space dimensions, Indiana Univ. Math J. 22 (1972), 101-134.

9. R. S. Phillips and L. Sarason, Energy decay for the neutrino equation in the exterior of a torus, Arch. Rational Mech. Anal. 41 (1971), 1-17. MR 43 \# 738.

10. J. R. Schulenberger and C. H. Wilcox, Eigenfunction expansions and scattering theory for wave propagation problems of classical physics, Arch. Rational Mech. Anal. 46 (1972), 280-320.

11. H. F. Walker, On the null-spaces of first-order elliptic partial differential operators in $R^{n}$, Proc. Amer. Math. Soc. 30 (1971), 278-286. MR 43 \#6583.

Department of Mathematics, University of California, Los Angeles, California 90024 